九州大学学術情報リポジトリ

Kyushu University Institutional Repository

\title{
Double zeta values, double Eisenstein series, and modular forms of level 2
}

Kaneko, Masanobu

Department of Mathematics, Faculty of Mathematics, Kyushu University : Professor

Tasaka, Koji

Graduate School of Mathematics, Kyushu University

http://hdl. handle. net/2324/27531

出版情報: Mathematische Annalen. 357 (3)，pp.1091-1118，2013-11-01. Springer Berlin Heidelberg バージョン：

権利関係 : 


\title{
Double zeta values, double Eisenstein series, and modular forms of level 2
}

\author{
Masanobu Kaneko · Koji Tasaka
}

Received: date / Accepted: date

\begin{abstract}
We study the double shuffle relations satisfied by the double zeta values of level 2, and introduce the double Eisenstein series of level 2 which satisfy the double shuffle relations. We connect the double Eisenstein series to modular forms of level 2.
\end{abstract}

Mathematics Subject Classification (2000) $11 \mathrm{M} 32 \cdot 11 \mathrm{~F} 11$

\section{Introduction}

In [7], H. Gangl, D. Zagier and the first author studied in detail the "double shuffle relations" satisfied by the double zeta values

$$
\zeta(r, s)=\sum_{m>n>0} \frac{1}{m^{r} n^{s}} \quad(r \geq 2, s \geq 1),
$$

and revealed in particular various connections between the space of double zeta values and the space of modular forms as well as their period polynomials on the full modular group $\operatorname{PSL}_{2}(\mathbf{Z})$. They also defined the "double Eisenstein series" and deduced the double shuffle relations for them, and in [9] we illustrated a way to connect the double Eisenstein series to the period polynomials of modular forms (of level 1).

This work is partially supported by Japan Society for the Promotion of Science, Grant-in-Aid for Scientific Research (S) 19104002, (B) 23340010.

Masanobu Kaneko

744, Motooka, Nishi-ku, Fukuoka, 819-0395, Japan.

Tel.: +81-92-802-4443

Fax: ++81-92-802-4405

E-mail: mkaneko@math.kyushu-u.ac.jp

Koji Tasaka

744, Motooka, Nishi-ku, Fukuoka, 819-0395, Japan.

Tel.: +81-92-802-4402

Fax: ++81-92-802-4405

E-mail: k-tasaka@math.kyushu-u.ac.jp 
In the present paper, we consider the double shuffle relations of level 2 and study the formal double zeta space, whose generators are the formal symbols corresponding to the double zeta values of level 2 (Euler sums) and the defining relations are the double shuffle relations. One of the relations we obtain in the formal double zeta space (Theorem 1) has an interesting application to the problem of representations of integers as sums of squares, and this will be given in the subsequent paper by the second author [12]. We then proceed to define the double Eisenstein series of level 2 and show that they also satisfy the double shuffle relations (Theorem 3), and have connections like in the case of level 1 to double zeta values, modular forms, and period polynomials, of level 2 (Theorem 5 and Corollary 1).

\section{The double zeta values of level 2}

The double zeta values of level 2 we are referring to are the following four types of real numbers given for integers $r \geq 2$ and $s \geq 1$ :

$$
\begin{gathered}
\zeta^{\mathbf{e e}}(r, s)=\sum_{\substack{m>n>0 \\
m, n \text { :even }}} \frac{1}{m^{r} n^{s}}, \quad \zeta^{\mathbf{e o}}(r, s)=\sum_{\substack{m>n>0 \\
m: \text { even }, n \text { :odd }}} \frac{1}{m^{r} n^{s}}, \\
\zeta^{\mathbf{o e}}(r, s)=\sum_{\substack{m>n>0 \\
m: \text { odd } n \text { : even }}} \frac{1}{m^{r} n^{s}}, \quad \zeta^{\mathbf{o o}}(r, s)=\sum_{\substack{m>n>0 \\
m, n \text { :odd }}} \frac{1}{m^{r} n^{s}} .
\end{gathered}
$$

These numbers can be written as simple linear combinations of the original multiple zeta values (1) and the numbers often referred to as Euler sums defined by

$$
\zeta(r, \bar{s})=\sum_{m>n>0} \frac{(-1)^{n}}{m^{r} n^{s}}, \zeta(\bar{r}, s)=\sum_{m>n>0} \frac{(-1)^{m}}{m^{r} n^{s}}, \zeta(\bar{r}, \bar{s})=\sum_{m>n>0} \frac{(-1)^{m+n}}{m^{r} n^{s}},
$$

and vice versa. Explicitly, we have the relations

$$
\left(\begin{array}{l}
\zeta^{\mathbf{e e}}(r, s) \\
\zeta^{\mathbf{e e}}(r, s) \\
\zeta^{\mathbf{e o}}(r, s) \\
\zeta^{\mathbf{o o}}(r, s)
\end{array}\right)=\frac{1}{4}\left(\begin{array}{cccc}
1 & 1 & 1 & 1 \\
1 & -1 & 1 & -1 \\
1 & 1 & -1 & -1 \\
1 & -1 & -1 & 1
\end{array}\right)\left(\begin{array}{l}
\zeta(r, s) \\
\zeta(\bar{r}, s) \\
\zeta(r, \bar{s}) \\
\zeta(\bar{r}, \bar{s})
\end{array}\right) .
$$

(The matrix on the right is invertible.) Note that, from the obvious relation

$$
\zeta(r, s)=\zeta^{\mathbf{e e}}(r, s)+\zeta^{\mathbf{e o}}(r, s)+\zeta^{\mathbf{o e}}(r, s)+\zeta^{\mathbf{o o}}(r, s)
$$

and

we have the relation

$$
\zeta(r, s)=2^{r+s} \zeta^{\mathbf{e e}}(r, s),
$$

$$
\left(2^{r+s}-1\right) \zeta^{\mathbf{e e}}(r, s)=\zeta^{\mathbf{e o}}(r, s)+\zeta^{\mathbf{o e}}(r, s)+\zeta^{\mathbf{o o}}(r, s) .
$$

We shall hereafter only consider $\zeta^{\mathbf{e o}}(r, s), \zeta^{\mathbf{e}}(r, s)$, and $\zeta^{\mathbf{o o}}(r, s)$. Moreover define

$$
\zeta^{\mathbf{e}}(k)=\sum_{n>0, \text { even }} \frac{1}{n^{k}} \quad \text { and } \quad \zeta^{\mathbf{o}}(k)=\sum_{n>0, \text { odd }} \frac{1}{n^{k}} \text {. }
$$

Then in the standard manner we can show the following double shuffle relations. 
Proposition 1 For positive integers $r, s \geq 2$, we have

$$
\begin{aligned}
& \zeta^{\mathbf{o}}(r) \zeta^{\mathbf{e}}(s)=\zeta^{\mathbf{o}}(r, s)+\zeta^{\mathbf{e}}(s, r)=\sum_{\substack{i+j=r+s \\
i \geq 2, j \geq 1}}\left(\left(\begin{array}{c}
i-1 \\
r-1
\end{array}\right) \zeta^{\mathbf{o}}(i, j)+\left(\begin{array}{c}
i-1 \\
s-1
\end{array}\right) \zeta^{\mathbf{o o}}(i, j)\right), \\
& \zeta^{\mathbf{o}}(r) \zeta^{\mathbf{o}}(s)=\zeta^{\mathbf{0 o}}(r, s)+\zeta^{\mathbf{o o}}(s, r)+\zeta^{\mathbf{o}}(r+s)=\sum_{\substack{i+j=r+s \\
i \geq 2, j \geq 1}}\left(\left(\begin{array}{c}
i-1 \\
r-1
\end{array}\right)+\left(\begin{array}{c}
i-1 \\
s-1
\end{array}\right)\right) \zeta^{\mathbf{e o}}(i, j) .
\end{aligned}
$$

Proof The first equality in each sequence of identities is obtained as usual from the manipulation of the defining series. For the second, we use the following integral representations of each zeta value and the shuffle product of integrals:

$$
\begin{aligned}
& \zeta^{\mathbf{o}}(k)=\int_{1>t_{1}>t_{2}>\cdots>t_{k}>0} \cdots \int_{t_{1}} \frac{d t_{1}}{t_{2}} \cdots \frac{d t_{k-1}}{t_{k-1}} \cdot \frac{d t_{k}}{1-t_{k}^{2}}, \\
& \zeta^{\mathbf{e}}(k)=\int_{1>t_{1}>t_{2}>\cdots>t_{k}>0} \ldots \int_{t_{1}} \frac{d t_{1}}{t_{2}} \cdots \frac{d t_{k-1}}{t_{k-1}} \cdot \frac{t_{k} d t_{k}}{1-t_{k}^{2}}, \\
& \zeta^{\mathbf{e o}}(r, s)=\int_{1>t_{1}>t_{2}>\cdots>t_{r+s}>0} \cdots \int_{t_{1}} \frac{d t_{1}}{t_{2}} \cdots \frac{d t_{2}}{t_{r-1}} \cdot \frac{d t_{r}}{1-t_{r}^{2}} \cdot \frac{d t_{r+1}}{t_{r+1}} \cdots \frac{d t_{r+s-1}}{t_{r+s-1}} \cdot \frac{d t_{r+s}}{1-t_{r+s}^{2}}, \\
& \zeta^{\mathbf{o e}}(r, s)=\int_{1>t_{1}>t_{2}>\cdots>t_{r+s}>0} \ldots \int_{t_{1}} \frac{d t_{1}}{t_{2}} \cdots \frac{d t_{2}}{t_{r-1}} \cdot \frac{d t_{r}}{1-t_{r}^{2}} \cdot \frac{d t_{r+1}}{t_{r+1}} \cdots \frac{d t_{r+s-1}}{t_{r+s-1}} \cdot \frac{t_{r+s} d t_{r+s}}{1-t_{r+s}^{2}}, \\
& \zeta^{\mathbf{0 o}}(r, s)=\int_{1>t_{1}>t_{2}>\cdots>t_{r+s}>0} \cdots \int_{t_{1}} \frac{d t_{1}}{t_{2}} \cdots \frac{d t_{2}}{t_{r-1}} \cdot \frac{t_{r} d t_{r}}{1-t_{r}^{2}} \cdot \frac{d t_{r+1}}{t_{r+1}} \cdots \frac{d t_{r+s-1}}{t_{r+s-1}} \cdot \frac{d t_{r+s}}{1-t_{r+s}^{2}} .
\end{aligned}
$$

The first two are easy to deduce, and to see the rest for double zetas, we use the expression (2) of each double zeta value in terms of Euler sums and the standard integral representations

$$
\begin{aligned}
& \zeta(r, s)=\int_{1>t_{1}>t_{2}>\cdots>t_{r+s}>0} \ldots \int_{t_{1}} \frac{d t_{1}}{t_{r-1}} \cdot \frac{d t_{r-1}}{1-t_{r}} \cdot \frac{d t_{r+1}}{t_{r+1}} \cdots \frac{d t_{r+s-1}}{t_{r+s-1}} \cdot \frac{d t_{r+s}}{1-t_{r+s}}, \\
& \zeta(\bar{r}, s)=\int_{1>t_{1}>t_{2}>\cdots>t_{r+s}>0} \cdots \int_{t_{1}} \frac{d t_{1}}{t_{r-1}} \cdot \frac{\left.d t_{r-1}\right)}{1+t_{r}} \cdot \frac{d t_{r+1}}{t_{r+1}} \cdots \frac{d t_{r+s-1}}{t_{r+s-1}} \cdot \frac{\left(-d t_{r+s}\right)}{1+t_{r+s}}, \\
& \zeta(r, \bar{s})=\int_{1>t_{1}>t_{2}>\cdots>t_{r+s}>0} \cdots \int_{1>t_{1}>t_{2}>\cdots>t_{r+s}>0} \frac{d t_{1}}{t_{1}} \cdots \frac{d t_{r-1}}{t_{r-1}} \cdot \frac{d t_{r}}{1-t_{r}} \cdot \frac{d t_{r+1}}{t_{r+1}} \cdots \frac{d t_{r+s-1}}{t_{r+s-1}} \cdot \frac{\left(-d t_{r+s}\right)}{1+t_{r+s}}, \\
& \zeta(\bar{r}, \bar{s})=\int_{1} \ldots \int_{t_{1}} \frac{d t_{r-1}}{t_{r-1}} \cdot \frac{\left(-d t_{r}\right)}{1+t_{r}} \cdot \frac{d t_{r+1}}{t_{r+1}} \cdots \frac{d t_{r+s-1}}{t_{r+s-1}} \cdot \frac{d t_{r+s}}{1-t_{r+s}} .
\end{aligned}
$$

Noting the identities

$$
\frac{1}{1-t^{2}}=\frac{1}{2}\left(\frac{1}{1-t}-\frac{(-1)}{1+t}\right), \quad \frac{t}{1-t^{2}}=\frac{1}{2}\left(\frac{1}{1-t}+\frac{(-1)}{1+t}\right)
$$


we obtain the desired integral expressions and hence the proposition by shuffle products of integrals.

Now we introduce the level 2 version of the formal double zeta space studied in [7] as follows. Let $k>2$ and $\mathscr{D}_{\mathscr{Z}_{k}}$ be the $\mathbf{Q}$-vector space spanned by formal symbols $Z_{r, s}^{\mathbf{e o}}, Z_{r, s}^{\mathbf{o e}}, Z_{r, s}^{\mathbf{o o}}, P_{r, s}^{\mathbf{o e}}, P_{r, s}^{\mathbf{o o}}(r, s \geq 1, r+s=k)$, and $Z_{k}^{\mathbf{0}}$ with the set of relations

$$
\begin{aligned}
& P_{r, s}^{\mathbf{o e}}=Z_{r, s}^{\mathbf{o e}}+Z_{s, r}^{\mathbf{e o}}=\sum_{\substack{i+j=k \\
i, j \geq 1}}\left(\left(\begin{array}{c}
i-1 \\
r-1
\end{array}\right) Z_{i, j}^{\mathbf{o e}}+\left(\begin{array}{c}
i-1 \\
s-1
\end{array}\right) Z_{i, j}^{\mathbf{0 o}}\right), \\
& P_{r, s}^{\mathbf{0 o}}=Z_{r, s}^{\mathbf{0 o}}+Z_{s, r}^{\mathbf{0 o}}+Z_{k}^{\mathbf{o}}=\sum_{\substack{i+j=k \\
i, j \geq 1}}\left(\left(\begin{array}{c}
i-1 \\
r-1
\end{array}\right)+\left(\begin{array}{c}
i-1 \\
s-1
\end{array}\right)\right) Z_{i, j}^{\mathbf{e o}}
\end{aligned}
$$

for $r, s \geq 1, r+s=k$, so that

$$
\mathscr{D} \mathscr{Z}_{k}=\frac{\left\{\mathbf{Q} \text {-linear combinations of } Z_{r, s}^{\mathbf{e o}}, Z_{r, s}^{\mathbf{o e}}, Z_{r, s}^{\mathbf{o o}}, P_{r, s}^{\mathbf{o e}}, P_{r, s}^{\mathbf{o o}}, Z_{k}^{\mathbf{o}}\right\}}{\langle\mathbf{Q} \text {-linear span of relations (3), (4) }\rangle} .
$$

Since the elements $P_{r, s}^{\mathbf{o e}}$ and $P_{r, s}^{\mathbf{o o}}$ are written in $Z$ 's, we can also regard the space as given by

$$
\mathscr{D} \mathscr{Z}_{k}=\frac{\left\{\mathbf{Q} \text {-linear combinations of } Z_{r, s}^{\mathbf{e o}}, Z_{r, s}^{\mathbf{o e}}, Z_{r, s}^{\mathbf{o o}}, Z_{k}^{\mathbf{o}}\right\}}{\langle\mathbf{Q} \text {-linear span of relations (5), (6) }\rangle}
$$

where the defining relations (5) and (6) are

$$
\begin{aligned}
& Z_{r, s}^{\mathbf{o e}}+Z_{s, r}^{\mathbf{e o}}=\sum_{\substack{i+j=k \\
i, j \geq 1}}\left(\left(\begin{array}{c}
i-1 \\
r-1
\end{array}\right) Z_{i, j}^{\mathbf{o e}}+\left(\begin{array}{c}
i-1 \\
s-1
\end{array}\right) Z_{i, j}^{\mathbf{o o}}\right), \\
& Z_{r, s}^{\mathbf{0 o}}+Z_{s, r}^{\mathbf{0 o}}+Z_{k}^{\mathbf{o}}=\sum_{\substack{i+j=k \\
i, j \geq 1}}\left(\left(\begin{array}{c}
i-1 \\
r-1
\end{array}\right)+\left(\begin{array}{c}
i-1 \\
s-1
\end{array}\right)\right) Z_{i, j}^{\mathbf{e o}} .
\end{aligned}
$$

Note that the relations (3) and (4) (as well as (5) and (6)) correspond to those in Proposition 1 when $r, s \geq 2$, under the correspondences

$$
\begin{gathered}
Z_{r, s}^{\mathbf{e o}} \longleftrightarrow \zeta^{\mathbf{e o}}(r, s), Z_{r, s}^{\mathbf{o e}} \longleftrightarrow \zeta^{\mathbf{o e}}(r, s), Z_{r, s}^{\mathbf{o o}} \longleftrightarrow \zeta^{\mathbf{0 o}}(r, s), Z_{k}^{\mathbf{o}} \longleftrightarrow \zeta^{\mathbf{o}}(k), \\
P_{r, s}^{\mathbf{o e}} \longleftrightarrow \zeta^{\mathbf{o}}(r) \zeta^{\mathbf{e}}(s), P_{r, s}^{\mathbf{0 o}} \longleftrightarrow \zeta^{\mathbf{o}}(r) \zeta^{\mathbf{o}}(s),
\end{gathered}
$$

because in that case the binomial coefficients for $i=1$ on the right vanishes. For our later applications it is convenient to allow the "divergent" $Z_{1, k-1}^{\mathbf{e o}}, P_{1, k-1}^{\mathbf{o e}}$ etc., and in fact the double shuffle relations in Proposition 1 can be extended for $r=1$ or $s=1$ by using a suitable regularization procedure for $\zeta(1, \bar{s})$ etc. developed in [2] (the case of $m=2$ in their notation). Specifically, by setting

$$
\zeta^{\mathbf{o}}(1):=\frac{1}{2}(T+\log 2), \zeta^{\mathbf{e}}(1):=\frac{1}{2}(T-\log 2)
$$


and, for $s \geq 2$

$$
\begin{aligned}
& \zeta^{\mathbf{e o}}(1, s)=\frac{1}{2} \zeta^{\mathbf{o}}(s) T-\frac{1}{2}(\log 2) \zeta^{\mathbf{o}}(s)-\zeta^{\mathbf{o}}(s, 1), \\
& \zeta^{\mathbf{0}}(1, s)=\frac{1}{2} \zeta^{\mathbf{e}}(s) T+\frac{1}{2}(\log 2) \zeta^{\mathbf{e}}(s)-\zeta^{\mathbf{e o}}(s, 1), \\
& \zeta^{\mathbf{o o}}(1, s)=\frac{1}{2} \zeta^{\mathbf{o}}(s) T+\frac{1}{2}(\log 2) \zeta^{\mathbf{o}}(s)-\zeta^{\mathbf{o o}}(s, 1)-\zeta^{\mathbf{o}}(s+1)
\end{aligned}
$$

where $T$ is a formal variable, the equations in Proposition 1 are valid for all $r, s \geq 1$ except $(r, s)=(1,1)$.

Theorem 1 Suppose $k$ is even and $k \geq 4$. In $\mathscr{D} \mathscr{Z}_{k}$, we have

1)

$$
\sum_{\substack{r=2 \\ r: \text { even }}}^{k-2} Z_{r, k-r}^{\mathbf{o o}}=\frac{1}{4} Z_{k}^{\mathbf{o}} .
$$

2) Each $P_{r, k-r}^{\mathbf{o e}}$ with $r$ even can be written as a $\mathbf{Q}$-linear combination of $P_{i, j}^{\mathbf{o o}}(i, j$ : even, $i+j=k$ ) and $Z_{k}^{\mathbf{0}}$

Proof Consider the generating functions

$$
\begin{aligned}
& \mathscr{Z}_{k}^{\mathbf{e o}}(X, Y)=\sum_{r+s=k} Z_{r, s}^{\mathbf{e o}} X^{r-1} Y^{s-1}, \mathscr{Z}_{k}^{\mathbf{o e}}(X, Y)=\sum_{r+s=k} Z_{r, s}^{\mathbf{o e}} X^{r-1} Y^{s-1}, \\
& \mathscr{Z}_{k}^{\mathbf{o o}}(X, Y)=\sum_{r+s=k} Z_{r, s}^{\mathbf{o}} X^{r-1} Y^{s-1} .
\end{aligned}
$$

Here and in the following, the sum $\sum_{r+s=k}$ always means $\sum_{r+s=k, r, s \geq 1}$. The double shuffle relations (5) and (6) are equivalent to the relations

$$
\begin{aligned}
& \mathscr{Z}_{k}^{\mathbf{o e}}(X, Y)+\mathscr{Z}_{k}^{\mathbf{e o}}(Y, X)=\mathscr{Z}_{k}^{\mathbf{o e}}(X+Y, Y)+\mathscr{Z}_{k}^{\mathbf{0 o}}(X+Y, X), \\
& \mathscr{Z}_{k}^{\mathbf{0 o}}(X, Y)+\mathscr{Z}_{k}^{\mathbf{0 o}}(Y, X)+Z_{k}^{\mathbf{0}} \cdot \frac{X^{k-1}-Y^{k-1}}{X-Y}=\mathscr{Z}_{k}^{\mathbf{e o}}(X+Y, Y)+\mathscr{Z}_{k}^{\mathbf{e o}}(X+Y, X) .
\end{aligned}
$$

Substituting $X=1, Y=0$ in (8) and $X=1, Y=-1$ in (9), we respectively obtain

$$
\begin{aligned}
& Z_{k-1,1}^{\mathbf{o e}}+Z_{1, k-1}^{\mathbf{e o}}=Z_{k-1,1}^{\mathbf{o e}}+\sum_{r=1}^{k-1} Z_{r, k-r}^{\mathbf{o o}}, \\
& 2 \sum_{r=1}^{k-1}(-1)^{r-1} Z_{r, k-r}^{\mathbf{0 o}}+Z_{k}^{\mathbf{o}}=2 Z_{1, k-1}^{\mathbf{e o}} .
\end{aligned}
$$

We divide (11) by 2 and add (10) to obtain

$$
\frac{1}{2} Z_{k}^{\mathbf{o}}=2 \sum_{\substack{r=2 \\ r: \text { even }}}^{k-2} Z_{r, k-r}^{\mathbf{o o}}
$$

and hence 1) of Theorem.

To prove 2), we need the following lemma. 
Lemma 1 Let $k \geq 4$ be an even integer and $a_{i, j}, b_{i, j}, c_{i, j}$ be rational numbers. Then the following two statements are equivalent.

1) The relation

$$
\sum_{i+j=k} a_{i, j} Z_{i, j}^{\mathbf{e o}}+\sum_{i+j=k} b_{i, j} Z_{i, j}^{\mathbf{o e}}+\sum_{i+j=k} c_{i, j} Z_{i, j}^{\mathbf{o o}} \equiv 0 \quad\left(\bmod \mathbf{Q} Z_{k}^{\mathbf{o}}\right)
$$

holds in $\mathscr{D}_{\mathcal{Z}}$ (as before $\sum_{i+j=k}$ means $\sum_{i+j=k, i, j \geq 1}$ ).

2) There exist some homogeneous polynomials $F, G \in \mathbf{Q}[X, Y]$ of degree $k-2$ such that

$$
\begin{aligned}
& F\left(Y_{1}, X_{1}\right)+F\left(X_{2}, Y_{2}\right)-F\left(X_{2}, X_{2}+Y_{2}\right)-F\left(X_{3}+Y_{3}, X_{3}\right) \\
& +G\left(X_{3}, Y_{3}\right)+G\left(Y_{3}, X_{3}\right)-G\left(X_{1}, X_{1}+Y_{1}\right)-G\left(X_{1}+Y_{1}, X_{1}\right) \\
& =\sum_{i+j=k}\left(\begin{array}{c}
k-2 \\
i-1
\end{array}\right) a_{i, j} X_{1}^{i-1} Y_{1}^{j-1}+\sum_{i+j=k}\left(\begin{array}{c}
k-2 \\
i-1
\end{array}\right) b_{i, j} X_{2}^{i-1} Y_{2}^{j-1}+\sum_{i+j=k}\left(\begin{array}{c}
k-2 \\
i-1
\end{array}\right) c_{i, j} X_{3}^{i-1} Y_{3}^{j-1} .
\end{aligned}
$$

Proof This is an analogue of Proposition 5.1 in [7]. Take $F(X, Y)=\left(\begin{array}{l}k-2 \\ r-1\end{array}\right) X^{r-1} Y^{s-1}$ (and $G=0$ ) and compute the coefficients of $F\left(Y_{1}, X_{1}\right)+F\left(X_{2}, Y_{2}\right)-F\left(X_{2}, X_{2}+Y_{2}\right)-$ $F\left(X_{3}+Y_{3}, X_{3}\right)$ using binomial theorem. Then the relation in 1) is exactly (not only $\bmod \mathbf{Q} Z_{k}^{\mathbf{0}}$ but as an exact equality) the relation (5). Similarly, by taking $G(X, Y)=$ $\left(\begin{array}{c}k-2 \\ r-1\end{array}\right) X^{r-1} Y^{s-1}$ (and $F=0$ ) and computing the coefficients of $G\left(X_{3}, Y_{3}\right)+G\left(Y_{3}, X_{3}\right)-$ $G\left(X_{1}, X_{1}+Y_{1}\right)-G\left(X_{1}+Y_{1}, X_{1}\right)$, we see that the relation in 1$)$ is the relation (6) modulo $\mathbf{Q} Z_{k}^{\mathbf{0}}$. Since any relation of the form in 1) in $\mathscr{D}_{\mathcal{Z}}$ should come from a linear combination of (5) and (6) modulo $\mathbf{Q} Z_{k}^{\mathbf{0}}$, and any homogeneous polynomial is a linear combination of monomials, we obtain the lemma.

Using the lemma, we are going to produce enough relations of the form

$$
\sum_{\substack{r+s=k \\ r, s: \text { even }}} \alpha_{r, s} P_{r, s}^{\mathbf{o e}} \equiv \sum_{\substack{r+s=k \\ r, s: \text { even }}} \beta_{r, s} P_{r, s}^{\mathbf{o o}} \quad\left(\bmod \mathbf{Q} Z_{k}^{\mathbf{o}}\right)
$$

such that we can solve these in $P_{r, s}^{\mathbf{o e}}$. In view of the relations

$$
P_{r, s}^{\mathbf{o e}}=Z_{r, s}^{\mathbf{o e}}+Z_{s, r}^{\mathbf{e o}}, P_{r, s}^{\mathbf{o o}} \equiv Z_{r, s}^{\mathbf{o o}}+Z_{s, r}^{\mathbf{o o}} \quad\left(\bmod \mathbf{Q} Z_{k}^{\mathbf{o}}\right)
$$

and the lemma, we obtain the relation of the form (12) if we can take $F$ and $G$ in 2) of Lemma 1 so that the coefficients satisfy

(i) $a_{i, j}=b_{j, i}$,

(ii) $c_{i, j}=c_{j, i}$,

(iii) $a_{i, j}=b_{i, j}=c_{i, j}=0$ for all odd $i, j$.

We now work for convenience with inhomogeneous polynomials. Recall the usual correspondences $f(x)=F(x, 1)$ and $F(X, Y)=Y^{k-2} f(X / Y)$, and the action of the group $\Gamma=\mathrm{PGL}_{2}(\mathbf{Z})$ on the space of polynomials of degree at most $k-2$ by (we are assuming $k$ is even)

$$
\left.f(x)\right|_{k-2}\left(\begin{array}{ll}
a & b \\
c & d
\end{array}\right)=(c x+d)^{k-2} f\left(\frac{a x+b}{c x+d}\right) .
$$


We extend this action to the group ring $\mathbf{Z}[\Gamma]$ by linearity. Set

$$
T=\left(\begin{array}{ll}
1 & 1 \\
0 & 1
\end{array}\right), S=\left(\begin{array}{cc}
0 & -1 \\
1 & 0
\end{array}\right), \varepsilon=\left(\begin{array}{cc}
-1 & 0 \\
0 & 1
\end{array}\right), \delta=\left(\begin{array}{ll}
0 & 1 \\
1 & 0
\end{array}\right) .
$$

Then the left-hand side of the equation in 2) of Lemma 1 can be written in inhomogeneous form as

$$
(f|\delta-g|(T S T+T S \varepsilon))\left(x_{1}\right)+(f \mid(1-T S T))\left(x_{2}\right)-(f|T S \varepsilon-g|(1+\delta))\left(x_{3}\right) .
$$

(We write $\mid$ instead of $\left.\right|_{k-2}$.)

Lemma 2 Suppose the polynomial $f(x)$ (of degree at most $k-2$ ) satisfies $f \mid T S T \varepsilon=$ $f$ and put $g=\frac{1}{2} f \mid T \varepsilon$. Then the expression (15) gives the coefficients (in Lemma 1-2) satisfying the above three conditions (i), (ii), (iii).

Proof Inserting $g=\frac{1}{2} f \mid T \varepsilon$ into (15) and using the assumption $f \mid T S T \varepsilon=f$, which is equivalent to $f|T S=f| T \varepsilon$ since $(T \varepsilon)^{2}=1$, and also using the identities TSTST= $S, T \varepsilon T=\varepsilon, \varepsilon S=\delta, \delta \varepsilon=\varepsilon \delta=S$ in $\Gamma$, we can write (15) as

$$
(f \mid \delta(1-\varepsilon))\left(x_{1}\right)+(f \mid(1-\varepsilon))\left(x_{2}\right)-(f \mid T(1-\varepsilon))\left(x_{3}\right) .
$$

Now the condition (iii) (the polynomial is even) is clear from this (being killed by $1+$ $\varepsilon$ ), and the conditions (i) and (ii) are respectively the consequences of the equations

$$
\begin{aligned}
f \mid \delta(1-\varepsilon) \delta & =f \mid(1-\varepsilon), \\
f \mid T(1-\varepsilon) \delta & =f|T \delta-f| T S=f|T \varepsilon S-f| T \varepsilon=f \mid T(1-\varepsilon) .
\end{aligned}
$$

Noting $T S T \varepsilon=\left(\begin{array}{cc}-1 & 0 \\ -1 & 1\end{array}\right)$ and hence

$$
(-x+1)\left(\frac{-x}{-x+1}\right)=-x, \text { and }(-x+1)\left(\frac{-x}{-x+1}-2\right)=x-2,
$$

we see that the polynomials $x^{r}(x-2)^{k-2-r}$ for $r=0,2, \ldots, k-2$ (even) satisfy the condition $f \mid T S T \varepsilon=f$ in Lemma 2. With this choice of $f$ (for $r=0,2, \ldots, k-4$ ) and $g$ in Lemma 2, we compute the coefficients in Lemma 1 by noting (13), (16) and by using

$$
\begin{aligned}
& x^{r}(x-2)^{k-2-r} \mid(1-\varepsilon)=x^{r}(x-2)^{k-2-r}-x^{r}(x+2)^{k-2-r} \\
& =-\sum_{\substack{i=1 \\
i: \text { odd }}}^{k-2-r-1}\left(\begin{array}{c}
k-2-r \\
i
\end{array}\right) 2^{k-1-r-i} x^{r+i} \\
& =-\sum_{\substack{i=r+2 \\
i: \text { even }}}^{k-2}\left(\begin{array}{c}
k-2-r \\
i-1-r
\end{array}\right) 2^{k-i} x^{i-1} \quad(r+i \rightarrow i-1) \\
& =-\left(\begin{array}{c}
k-2 \\
r
\end{array}\right)^{-1} \sum_{\substack{i=r+2 \\
i: \text { even }}}^{k-2}\left(\begin{array}{c}
k-2 \\
i-1
\end{array}\right)\left(\begin{array}{c}
i-1 \\
r
\end{array}\right) 2^{k-i} x^{i-1},
\end{aligned}
$$


to obtain a relation of the form

$$
\sum_{\substack{i=r+2 \\
i: \text { even }}}^{k-2}\left(\begin{array}{c}
i-1 \\
r
\end{array}\right) 2^{k-i} P_{i, k-i}^{\mathbf{o e}} \equiv \text { linear combination of } P_{\text {even,even }}^{\mathbf{o o}} \quad\left(\bmod \mathbf{Q} Z_{k}^{\mathbf{o}}\right)
$$

When we put $r=k-4, \ldots, 2,0$, we can solve these congruences successively in each $P_{i, k-i}^{\mathbf{o e}}$ for $i=k-2, k-4, \ldots, 2$ (because the system is triangular). This completes the proof of Theorem 1.

\section{The double Eisenstein series of level 2}

\subsection{Definition and the double shuffle relations}

We introduce the double Eisenstein series of level 2 and first show that they satisfy the double shuffle relations.

Let ev (resp. od) be the set of even (resp. odd) integers and $\tau$ a variable in the upper half-plane. Define the three double Eisenstein series $G_{r, s}^{\mathbf{e o}}(\tau), G_{r, s}^{\mathbf{o e}}(\tau)$, and $G_{r, s}^{\mathbf{0 o}}(\tau)$ by

$$
\begin{aligned}
& G_{r, s}^{\mathbf{e o}}(\tau):=(2 \pi i)^{-r-s} \sum_{\substack{\lambda>\mu>0 \\
\lambda \in \mathbf{e v} \cdot \tau+\mathbf{e v} \\
\mu \in \mathbf{e v} \cdot \tau+\mathbf{o d}}} \frac{1}{\lambda^{r} \mu^{s}}=(2 \pi i)^{-r-s} \sum_{\substack{m \tau+n>m^{\prime} \tau+n^{\prime}>0 \\
m \in \mathbf{e v}, n \in \mathbf{e v} \\
m^{\prime} \in \mathbf{e v}, n^{\prime} \in \mathbf{o d}}} \frac{1}{(m \tau+n)^{r}\left(m^{\prime} \tau+n^{\prime}\right)^{s}}, \\
& G_{r, s}^{\mathbf{o e}}(\tau):=(2 \pi i)^{-r-s} \sum_{\substack { \lambda>\mu>0 \\
\begin{subarray}{c}{\lambda \in \mathbf{e v} \cdot \tau+\mathbf{o d} \\
\mu \in \mathbf{e v} \cdot \tau+\mathbf{e v}{ \lambda > \mu > 0 \\
\begin{subarray} { c } { \lambda \in \mathbf { e v } \cdot \tau + \mathbf { o d } \\
\mu \in \mathbf { e v } \cdot \tau + \mathbf { e v } } }\end{subarray}} \frac{1}{\lambda^{r} \mu^{s}}, \quad G_{r, s}^{\mathbf{o o}}(\tau):=(2 \pi i)^{-r-s} \sum_{\substack{\lambda>\mu>0 \\
\lambda \in \mathbf{e v} \cdot \tau+\mathbf{o d} \\
\mu \in \mathbf{e v} \cdot \tau+\mathbf{o d}}} \frac{1}{\lambda^{r} \mu^{s}} .
\end{aligned}
$$

Here, the positivity $m \tau+n>0$ of a lattice point means either $m>0$ or $m=0, n>0$, and $m \tau+n>m^{\prime} \tau+n^{\prime}$ means $\left(m-m^{\prime}\right) \tau+\left(n-n^{\prime}\right)>0$. We assume $r \geq 3$ and $s \geq 2$ for the absolute convergence.

All the series in (17) is easily seen to be invariant the translation $\tau \rightarrow \tau+1$, and hence have Fourier expansions. The Fourier series developments can be deduced in a quite similar manner to the full modular case [7]. In particular, our double zeta values of level 2 appear as constant terms.

Theorem 2 Let $r \geq 3$ and $s \geq 2$ be integers and set $k=r+s$. We have the following $q$-series expansions $\left(q=e^{2 \pi i \tau}\right)$. 
Double zeta values, double Eisenstein series, and modular forms of level 2

9

$$
\begin{aligned}
& G_{r, s}^{\mathbf{e o}}(\tau)=\tilde{\zeta}^{\mathbf{e o}}(r, s)+g_{r, s}^{\mathbf{e o}}(q) \\
& +\sum_{\substack{p+h=k \\
p>1}}\left\{\left((-1)^{s}\left(\begin{array}{c}
p-1 \\
s-1
\end{array}\right)+\delta_{p, s}\right) \widetilde{\zeta}^{\mathbf{o}}(p) g_{h}^{\mathbf{e}}(q)+(-1)^{p+r}\left(\begin{array}{c}
p-1 \\
r-1
\end{array}\right) \widetilde{\zeta}^{\mathbf{o}}(p) g_{h}^{\mathbf{o}}(q)\right\}, \\
& G_{r, s}^{\mathbf{o e}}(\tau)=\widetilde{\zeta}^{\mathbf{o e}}(r, s)+g_{r, s}^{\mathbf{o e}}(q) \\
& +\sum_{\substack{p+h=k \\
p>1}}\left\{(-1)^{s}\left(\begin{array}{c}
p-1 \\
s-1
\end{array}\right) \widetilde{\zeta} \mathbf{o}(p) g_{h}^{\mathbf{o}}(q)+\delta_{p, s} \widetilde{\zeta}^{\mathbf{e}}(p) g_{h}^{\mathbf{o}}(q)+(-1)^{p+r}\left(\begin{array}{c}
p-1 \\
r-1
\end{array}\right) \widetilde{\zeta} \mathbf{o}(p) g_{h}^{\mathbf{e}}(q)\right\} \\
& G_{r, s}^{\mathbf{o o}}(\tau)=\widetilde{\zeta}^{\mathbf{o o}}(r, s)+g_{r, s}^{\mathbf{o o}}(q) \\
& +\sum_{\substack{p+h=k \\
p>1}}\left\{\left((-1)^{s}\left(\begin{array}{c}
p-1 \\
s-1
\end{array}\right)+(-1)^{p+r}\left(\begin{array}{c}
p-1 \\
r-1
\end{array}\right)\right) \widetilde{\zeta}^{\mathbf{e}}(p) g_{h}^{\mathbf{o}}(q)+\delta_{p, s} \widetilde{\zeta}^{\mathbf{o}}(p) g_{h}^{\mathbf{o}}(q)\right\},
\end{aligned}
$$

where $\delta_{p, s}$ is Kronecker's delta, $\widetilde{\zeta}^{* *}(r, s)=(2 \pi i)^{-r-s} \zeta^{* *}(r, s)$ and $\widetilde{\zeta}^{*}(k)=(2 \pi i)^{-k} \zeta^{*}(k)$ $(*=\mathbf{e}$ or $\mathbf{o})$, and the $g$ 's are the following $q$-series:

$$
\begin{aligned}
& g_{r, s}^{\mathbf{e o}}(q)=-\frac{(-1)^{r+s}}{2^{r+s}(r-1) !(s-1) !} \sum_{\substack{m>m^{\prime}>0 \\
u, v>0}}(-1)^{v} u^{r-1} v^{s-1} q^{u m+v m^{\prime}}, \\
& g_{r, s}^{\mathbf{o e}}(q)=-\frac{(-1)^{r+s}}{2^{r+s}(r-1) !(s-1) !} \sum_{\substack{m>m^{\prime}>0 \\
u, v>0}}(-1)^{u} u^{r-1} v^{s-1} q^{u m+v m^{\prime}}, \\
& g_{r, s}^{\mathbf{o o}}(q)=\frac{(-1)^{r+s}}{2^{r+s}(r-1) !(s-1) !} \sum_{\substack{m>m^{\prime}>0 \\
u, v>0}}(-1)^{u+v} u^{r-1} v^{s-1} q^{u m+v m^{\prime}},
\end{aligned}
$$

and

$$
g_{r}^{\mathbf{e}}(q)=\frac{(-1)^{r}}{2^{r}(r-1) !} \sum_{u, m>0} u^{r-1} q^{u m}, g_{r}^{\mathbf{o}}(q)=\frac{(-1)^{r}}{2^{r}(r-1) !} \sum_{u, m>0}(-1)^{u} u^{r-1} q^{u m} .
$$

Proof Put, for positive integers $r$ and $s$,

$$
\varphi_{r}^{\mathbf{e}}(q)=\frac{(-1)^{r}}{2^{r}(r-1) !} \sum_{u>0} u^{r-1} q^{u / 2}, \varphi_{r}^{\mathbf{o}}(q)=\frac{(-1)^{r}}{2^{r}(r-1) !} \sum_{u>0}(-1)^{u} u^{r-1} q^{u / 2}
$$

Then the series $g_{r}^{*}(q), g_{r, s}^{* *}(q)$ in the theorem can be written by using $\varphi_{r}^{*}(q)$ as

$$
\begin{aligned}
g_{r}^{\mathbf{e}}(q) & =\sum_{m>0} \varphi_{r}^{\mathbf{e}}\left(q^{2 m}\right), g_{r}^{\mathbf{o}}(q)=\sum_{m>0} \varphi_{r}^{\mathbf{o}}\left(q^{2 m}\right), \\
g_{r, s}^{\mathbf{e}}(q) & =\sum_{m>m^{\prime}>0} \varphi_{r}^{\mathbf{e}}\left(q^{2 m}\right) \varphi_{s}^{\mathbf{o}}\left(q^{2 m^{\prime}}\right), g_{r, s}^{\mathbf{o e}}(q)=\sum_{m>m^{\prime}>0} \varphi_{r}^{\mathbf{o}}\left(q^{2 m}\right) \varphi_{s}^{\mathbf{e}}\left(q^{2 m^{\prime}}\right), \\
g_{r, s}^{\mathbf{o o}}(q) & =\sum_{m>m^{\prime}>0} \varphi_{r}^{\mathbf{o}}\left(q^{2 m}\right) \varphi_{s}^{\mathbf{o}}\left(q^{2 m^{\prime}}\right), g_{r, s}^{\mathbf{e}}(q)=\sum_{m>m^{\prime}>0} \varphi_{r}^{\mathbf{e}}\left(q^{2 m}\right) \varphi_{s}^{\mathbf{e}}\left(q^{2 m^{\prime}}\right) .
\end{aligned}
$$


The computation of the Fourier series can be carried out in a completely similar fashion as done in [7], dividing the sum of the defining series into four terms, according as $m=m^{\prime}=0, m=m^{\prime}>0, m>m^{\prime}=0, m>m^{\prime}>0$. For instance, in the case of $G_{r, s}^{\mathbf{e o}}(\tau)$, we compute

$$
G_{r, s}^{\mathbf{e o}}(\tau)=\left\{\sum_{\substack{m=m^{\prime}=0 \\ n>n^{\prime}>0 \\ m, m^{\prime}, n \in \mathbf{e v} \\ n^{\prime} \in \mathbf{o d}}}+\sum_{\substack{m=m^{\prime}>0 \\ n>n^{\prime} \\ m, m^{\prime}, n \in \mathbf{e v} \\ n^{\prime} \in \mathbf{o d}}}+\sum_{\substack{m>m^{\prime}=0 \\ n^{\prime}>0 \\ m, m^{\prime}, n \in \mathbf{e v} \\ n^{\prime} \in \mathbf{o d}}}+\sum_{\substack{m>m^{\prime}>0 \\ m, m^{\prime}, n \in \mathbf{e v} \\ n^{\prime} \in \mathbf{o d}}}\right\} \frac{(2 \pi i)^{-r-s}}{(m \tau+n)^{r}\left(m^{\prime} \tau+n^{\prime}\right)^{s}}
$$

using the partial fraction decomposition

$$
\begin{aligned}
\frac{1}{(\tau+n)^{r}\left(\tau+n^{\prime}\right)^{s}}= & (-1)^{s} \sum_{i=0}^{r-1}\left(\begin{array}{c}
s+i-1 \\
i
\end{array}\right) \frac{1}{(\tau+n)^{r-i}} \cdot \frac{1}{\left(n-n^{\prime}\right)^{s+i}} \\
& +\sum_{j=0}^{s-1}(-1)^{j}\left(\begin{array}{c}
r+j-1 \\
j
\end{array}\right) \frac{1}{\left(\tau+n^{\prime}\right)^{s-j}} \cdot \frac{1}{\left(n-n^{\prime}\right)^{r+j}}
\end{aligned}
$$

and the formulas

$$
\begin{gathered}
\sum_{n \in \mathbf{Z}} \frac{1}{(\tau+2 n)^{l}}=\frac{(-2 \pi i)^{l}}{2^{l}(l-1) !} \sum_{u>0} u^{l-1} q^{u / 2}=(2 \pi i)^{l} \varphi_{l}^{\mathbf{e}}(q) \quad(l \geq 2) \\
\sum_{n \in \mathbf{Z}} \frac{1}{(\tau+2 n+1)^{l}}=\frac{(-2 \pi i)^{l}}{2^{l}(l-1) !} \sum_{u>0}(-1)^{u} u^{l-1} q^{u / 2}=(2 \pi i)^{l} \varphi_{l}^{\mathbf{o}}(q) \quad(l \geq 2)
\end{gathered}
$$

(consequences of the standard Lipschitz formula, and when $l=1$ we use

$$
\begin{aligned}
\lim _{N \rightarrow \infty} \sum_{n=-N}^{N} \frac{1}{\tau+2 n} & =-\frac{\pi i}{2}+\frac{(-2 \pi i)}{2} \sum_{u>0} q^{u / 2}=-\frac{\pi i}{2}+(2 \pi i) \varphi_{1}^{\mathbf{e}}(q), \\
\lim _{N \rightarrow \infty} \sum_{n=-N}^{N} \frac{1}{\tau+2 n+1} & =-\frac{\pi i}{2}+(2 \pi i) \varphi_{1}^{\mathbf{o}}(q)
\end{aligned}
$$

instead). We leave the details to the reader.

We remark that each series in Theorem 2 is in $\mathbf{R}+q \mathbf{Q}[[q]]+\sqrt{-1} \mathbf{R}[[q]]$, and the terms in $\sqrt{-1} \mathbf{R}[[q]]$ ("imaginary part") only come from the terms having $\widetilde{\zeta}^{*}(p)$ with odd $p$ as coefficients (note that $\widetilde{\zeta}^{*}(p)$ is in $\mathbf{Q}$ or $\sqrt{-1} \mathbf{R}$ according to the parity of $p$ ).

Now we extend the definition of the double Eisenstein series for any (non-converging) $r, s \geq 1$ (except $r=s=1$ ), by using $q$-series. For this, we separately define the imag- 
inary part and the "combinatorial part". First we define the imaginary parts as

$$
\begin{aligned}
& I_{r, s}^{\mathbf{e o}}(q)=\sum_{\substack{p+h=k \\
p: \text { odd }}}\left\{\left((-1)^{s}\left(\begin{array}{c}
p-1 \\
s-1
\end{array}\right)+\delta_{p, s}\right) \widetilde{\zeta}^{\mathbf{o}}(p) g_{h}^{\mathbf{e}}(q)+(-1)^{p+r}\left(\begin{array}{c}
p-1 \\
r-1
\end{array}\right) \widetilde{\zeta}^{\mathbf{o}}(p) g_{h}^{\mathbf{o}}(q)\right\}, \\
& I_{r, s}^{\mathbf{o}}(q)=\sum_{\substack{p+h=k \\
p: \text { odd }}}\left\{(-1)^{s}\left(\begin{array}{c}
p-1 \\
s-1
\end{array}\right) \widetilde{\zeta}^{\mathbf{o}}(p) g_{h}^{\mathbf{o}}(q)+\delta_{p, s} \widetilde{\zeta}^{\mathbf{e}}(p) g_{h}^{\mathbf{o}}(q)+(-1)^{p+r}\left(\begin{array}{c}
p-1 \\
r-1
\end{array}\right) \widetilde{\zeta}^{\mathbf{o}}(p) g_{h}^{\mathbf{e}}(q)\right\}, \\
& I_{r, s}^{\mathbf{o}}(q)=\sum_{\substack{p+h=k \\
p: \text { odd }}}\left\{\left((-1)^{s}\left(\begin{array}{c}
p-1 \\
s-1
\end{array}\right)+(-1)^{p+r}\left(\begin{array}{c}
p-1 \\
r-1
\end{array}\right)\right) \widetilde{\zeta}^{\mathbf{e}}(p) g_{h}^{\mathbf{o}}(q)+\delta_{p, s} \widetilde{\zeta}^{\mathbf{o}}(p) g_{h}^{\mathbf{o}}(q)\right\} .
\end{aligned}
$$

The sum is over $p, h \geq 1$ with $p$ odd. Note that the regularized values $\widetilde{\zeta}^{\mathbf{o}}(1)$ and $\widetilde{\zeta}^{\mathbf{e}}(1)$ are defined by (7) and thus for any positive integers $r, s$, these series are elements of $\sqrt{-1} \mathbf{R}[T][[q]]$. Secondly, we define the part in $q \mathbf{Q}[[q]]$ which is referred to as the combinatorial double Eisenstein series. Put

$$
\begin{aligned}
& \beta_{r, s}^{\mathbf{e o}}(q)=\sum_{p+h=r+s}\left\{\left((-1)^{s}\left(\begin{array}{l}
p-1 \\
s-1
\end{array}\right)+\delta_{p, s}\right) \beta_{p}^{\mathbf{o}} g_{h}^{\mathbf{e}}(q)+(-1)^{p+r}\left(\begin{array}{c}
p-1 \\
r-1
\end{array}\right) \beta_{p}^{\mathbf{o}} g_{h}^{\mathbf{o}}(q)\right\}, \\
& \beta_{r, s}^{\mathbf{o e}}(q)=\sum_{p+h=r+s}\left\{(-1)^{s}\left(\begin{array}{l}
p-1 \\
s-1
\end{array}\right) \beta_{p}^{\mathbf{o}} g_{h}^{\mathbf{o}}(q)+\boldsymbol{\delta}_{p, s} \beta_{p}^{\mathbf{e}} g_{h}^{\mathbf{o}}(q)+(-1)^{p+r}\left(\begin{array}{c}
p-1 \\
r-1
\end{array}\right) \beta_{p}^{\mathbf{o}} g_{h}^{\mathbf{e}}(q)\right\}, \\
& \beta_{r, s}^{\mathbf{o o}}(q)=\sum_{p+h=r+s}\left\{\left((-1)^{s}\left(\begin{array}{l}
p-1 \\
s-1
\end{array}\right)+(-1)^{p+r}\left(\begin{array}{l}
p-1 \\
r-1
\end{array}\right)\right) \beta_{p}^{\mathbf{e}} g_{h}^{\mathbf{o}}(q)+\delta_{p, s} \boldsymbol{\beta}_{p}^{\mathbf{o}} g_{h}^{\mathbf{o}}(q)\right\},
\end{aligned}
$$

where

$$
\beta_{r}^{\mathbf{e}}=-\frac{B_{r}}{2^{r+1} \cdot r !}, \beta_{r}^{\mathbf{o}}=-\frac{\left(1-2^{-r}\right) B_{r}}{2 \cdot r !} \quad\left(B_{r}=\text { the Bernoulli number }\right),
$$

and as before the condition " $p+h=r+s$ " includes " $p, h \geq 1$ ". Let

$$
\bar{g}_{r}^{\mathbf{e}}(q):=-\sum_{m>0} m \varphi_{r+1}^{\mathbf{e}}\left(q^{2 m}\right), \bar{g}_{r}^{\mathbf{o}}(q):=-\sum_{m>0} m \varphi_{r+1}^{\mathbf{o}}\left(q^{2 m}\right) \quad(r \geq 0),
$$

and for integers $r, s \geq 1$ let

$$
\begin{aligned}
& \varepsilon_{r, s}^{\mathbf{e o}}(q)=\delta_{r, 2} \bar{g}_{s}^{\mathbf{o}}(q)-\delta_{r, 1} \bar{g}_{s-1}^{\mathbf{o}}(q)+\delta_{s, 1}\left(\bar{g}_{r-1}^{\mathbf{e}}(q)+g_{r}^{\mathbf{e}}(q)\right)+\delta_{r, 1} \delta_{s, 1} \alpha_{1}, \\
& \varepsilon_{r, s}^{\mathbf{o e}}(q)=\delta_{r, 2} \bar{g}_{s}^{\mathbf{e}}(q)-\delta_{r, 1} \bar{g}_{s-1}^{\mathbf{e}}(q)+\delta_{s, 1}\left(\bar{g}_{r-1}^{\mathbf{o}}(q)+g_{r}^{\mathbf{o}}(q)\right)+\delta_{r, 1} \delta_{s, 1} \alpha_{2}, \\
& \varepsilon_{r, s}^{\mathbf{o o}}(q)=\delta_{r, 2} \bar{g}_{s}^{\mathbf{o}}(q)-\delta_{r, 1} \bar{g}_{s-1}^{\mathbf{o}}(q)+\delta_{s, 1}\left(\bar{g}_{r-1}^{\mathbf{o}}(q)+g_{r}^{\mathbf{o}}(q)\right)+\delta_{r, 1} \delta_{s, 1} \alpha_{3},
\end{aligned}
$$

where

$$
\alpha_{1}=\bar{g}_{0}^{\mathbf{0}}(q)-\frac{1}{2} \bar{g}_{0}^{\mathbf{e}}(q), \alpha_{2}=-\alpha_{1}, \alpha_{3}=4 g_{2}^{\mathbf{0}}(q)+\frac{1}{2} \bar{g}_{0}^{\mathbf{e}}(q) .
$$

Note that each $\varepsilon_{r, s}^{* * *}$ is 0 when $r \geq 3$ and $s \geq 2$, and in the other boundary cases, the definition is adopted (quite similarly as in [7]) so that the extended double Eisenstein 
series below satisfy the double shuffle relations. The combinatorial double Eisenstein series are then defined, for positive integers $r, s \geq 1$, by

$$
\begin{aligned}
& C_{r, S}^{\mathbf{e o}}(q)=g_{r, s}^{\mathbf{e}}(q)+\beta_{r, s}^{\mathbf{e o}}(q)+\frac{1}{4} \varepsilon_{r, s}^{\mathbf{e o}}(q), \\
& C_{r, S}^{\mathbf{e o}}(q)=g_{r, s}^{\mathbf{e}}(q)+\beta_{r, s}^{\mathbf{o e}}(q)+\frac{1}{4} \varepsilon_{r, s}^{\mathbf{e}}(q), \\
& C_{r, S}^{\mathbf{o o}}(q)=g_{r, S}^{\mathbf{o o}}(q)+\beta_{r, s}^{\mathbf{o o}}(q)+\frac{1}{4} \varepsilon_{r, s}^{\mathbf{o}}(q) .
\end{aligned}
$$

Lastly, the constant term of the double Eisenstein series is given by the (regularized) double zeta values.

Definition 1 For any integers $r, s \geq 1$ with $(r, s) \neq(1,1)$, we define

$$
\begin{aligned}
& G_{r, s}^{\mathbf{e}}(q):=\widetilde{\zeta}^{\mathbf{e}}(r, s)+C_{r, s}^{\mathbf{e o}}(q)+I_{r, s}^{\mathbf{e}}(q), \\
& G_{r, s}^{\mathbf{o e}}(q):=\widetilde{\zeta}^{\mathbf{o}}(r, s)+C_{r, s}^{\mathbf{o e}}(q)+I_{r, s}^{\mathbf{o e}}(q), \\
& G_{r, s}^{\mathbf{o o}}(q):=\widetilde{\zeta}^{\mathbf{o o}}(r, s)+C_{r, s}^{\mathbf{o o}}(q)+I_{r, s}^{\mathbf{o o}}(q) .
\end{aligned}
$$

To state the double shuffle relations in the forms (3) and (4) for these series, we need usual Eisenstein series for the congruence subgroup $\Gamma_{0}(2)=\left\{\left(\begin{array}{cc}a & b \\ c & d\end{array}\right) \in \mathrm{SL}_{2}(\mathbf{Z}) \mid c \equiv 0 \bmod 2\right\}$. For each integer $k \geq 3$, let the series $G_{k}^{(i \infty)}(\tau)$ and $G_{k}^{(0)}(\tau)$ be defined by

$$
G_{k}^{(i \infty)}(\tau):=\sum_{\substack{\lambda>0 \\ \lambda \in \mathbf{e v} \cdot \tau+\mathbf{o d}}} \frac{1}{\lambda^{k}}=\sum_{\substack{m \tau+n>0 \\ m: \text { even, }, \text { odd }}} \frac{1}{(m \tau+n)^{k}},
$$

and

$$
G_{k}^{(0)}(\tau):=\sum_{\substack{\lambda>0 \\ \lambda \in \mathbf{o d} \cdot \tau+\mathbf{Z}}} \frac{1}{\lambda^{k}}=\sum_{\substack{m \tau+n>0 \\ m: \text { odd }}} \frac{1}{(m \tau+n)^{k}} .
$$

When $k \geq 4$ is even, the functions $G_{k}^{(i \infty)}(\tau)$ and $G_{k}^{(0)}(\tau)$ are the Eisenstein series for $\Gamma_{0}(2)$ associated to cusps $i \infty$ and 0 respectively, and as such they are modular of weight $k$ with respect to $\Gamma_{0}(2)$. The Fourier series of $G_{k}^{(i \infty)}(\tau)$ and $G_{k}^{(0)}(\tau)$ are given as follows. Let $G_{k}(\tau)$ be the Eisenstein series of weight $k$ for $\mathrm{SL}_{2}(\mathbf{Z})$ :

$$
G_{k}(\tau):=\sum_{\mathbf{Z} \tau+\mathbf{Z} \ni m \tau+n>0} \frac{1}{(m \tau+n)^{k}}=\zeta(k)+\frac{(-2 \pi i)^{k}}{(k-1) !} \sum_{n \geq 1} \sigma_{k-1}(n) q^{n}, \quad\left(\sigma_{k-1}(n)=\sum_{d \mid n} d^{k-1}\right) .
$$

(Note that this gives a non-zero function even when $k$ is odd.) With this we have

$$
\begin{aligned}
G_{k}^{(i \infty)}(\tau) & =G_{k}(2 \tau)-2^{-k} G_{k}(\tau)=\zeta^{\mathbf{o}}(k)+\frac{(-2 \pi i)^{k}}{2^{k}(k-1) !} \sum_{n \geq 1}\left(\sum_{d \mid n}(-1)^{d} d^{k-1}\right) q^{n} \\
G_{k}^{(0)}(\tau) & =G_{k}(\tau)-G_{k}(2 \tau)=\frac{(-2 \pi i)^{k}}{(k-1) !} \sum_{n \geq 1}\left(\sum_{\substack{d \mid n \\
n / d \text { odd }}} d^{k-1}\right) q^{n}
\end{aligned}
$$


We define the $q$-series $G_{k}(q), G_{k}^{(i \infty)}(q)$, and $G_{k}^{(0)}(q)$ for any $k \geq 1$ by the (convergent) $q$-series on the right-hand sides of (20) and (21), with the regularized values (7) and $\zeta(1)=T\left(=\zeta^{\mathbf{e}}(1)+\zeta^{\mathbf{o}}(1)\right)$. Finally we set

$$
\begin{aligned}
& G_{k}^{\mathbf{o}}(q)=(2 \pi i)^{-k} G_{k}^{(i \infty)}(q)=\widetilde{\zeta}^{\mathbf{o}}(k)+g_{k}^{\mathbf{o}}(q), \\
& G_{k}^{\mathbf{e}}(q)=2^{-k}(2 \pi i)^{-k} G_{k}(q)=\widetilde{\zeta} \mathbf{e}(k)+g_{k}^{\mathbf{e}}(q) .
\end{aligned}
$$

Theorem 3 For any integers $r, s \geq 1$ with $(r, s) \neq(1,1)$, we have

$$
\begin{aligned}
G_{r}^{\mathbf{o}}(q) G_{s}^{\mathbf{e}}(q)+\frac{1}{4}\left(\delta_{r, 2} \bar{g}_{s}^{\mathbf{e}}(q)+\delta_{s, 2} \bar{g}_{r}^{\mathbf{o}}(q)\right) & =G_{r, s}^{\mathbf{o e}}(q)+G_{s, r}^{\mathbf{e o}}(q) \\
& =\sum_{i+j=r+s}\left(\left(\begin{array}{c}
i-1 \\
r-1
\end{array}\right) G_{i, j}^{\mathbf{e e}}(q)+\left(\begin{array}{c}
i-1 \\
s-1
\end{array}\right) G_{i, j}^{\mathbf{o o}}(q)\right), \\
G_{r}^{\mathbf{o}}(q) G_{s}^{\mathbf{o}}(q)+\frac{1}{4}\left(\delta_{r, 2} \bar{g}_{s}^{\mathbf{o}}(q)+\delta_{s, 2} \bar{g}_{r}^{\mathbf{o}}(q)\right) & =G_{r, s}^{\mathbf{o o}}(q)+G_{s, r}^{\mathbf{o o}}(q)+G_{r+s}^{\mathbf{o}}(q) \\
& =\sum_{i+j=r+s}\left(\left(\begin{array}{c}
i-1 \\
r-1
\end{array}\right)+\left(\begin{array}{c}
i-1 \\
s-1
\end{array}\right)\right) G_{i, j}^{\mathbf{e o}}(q) .
\end{aligned}
$$

The proof of the theorem will be postponed to $\S 3.3$.

\subsection{Double Eisenstein series and period polynomials}

In this subsection, we describe a mysterious connection between our double Eisenstein series and the period polynomials associated to cusp forms on $\Gamma_{0}(2)$. This kind of connection was first observed in the full modular case [9], which will be recalled briefly in the appendix for the convenience of the reader because the reference [9] circulated only among participants of the conference.

Let us recall the theory of period polynomials for $\Gamma_{0}(2)$ given in [8] and [6]. We follow the formulation of [10]. Recall the group $\Gamma_{0}(2)$ is generated by two elements (see e.g. [1, Theorem 4.3]))

$$
T=\left(\begin{array}{ll}
1 & 1 \\
0 & 1
\end{array}\right), M=\left(\begin{array}{cc}
-1 & -1 \\
2 & 1
\end{array}\right) .
$$

Let $k$ be a positive even integer and $V_{k}$ be the space of polynomials with rational coefficients of degree at most $k-2$ :

$$
V_{k}:=\{P(X) \in \mathbf{Q}[X] \mid \operatorname{deg}(f) \leq k-2\} .
$$

The group $\Gamma_{0}(2)$ acts on $V_{k}$ as in (14) and this action extends to that of the group ring $\mathbf{Z}\left[\Gamma_{0}(2)\right]$ as usual. Consider the subspace $W_{k}$ of $V_{k}$ defined by

$$
W_{k}:=\left\{P \in V_{k}|P|_{k-2}(1-T)(1+M)=0\right\} .
$$

For a cusp form $f \in S_{k}(2):=\left\{\right.$ the space of cusp forms on $\left.\Gamma_{0}(2)\right\}$, the period polynomial $r_{f}(X)$ is given by

$$
r_{f}(X):=\int_{0}^{i \infty} f(\tau)(X-\tau)^{n} d \tau
$$


It is implicitly shown in the proof of Proposition 3 in [8] that

$$
r_{f}(X) \in W_{k} \otimes \mathbf{C} .
$$

Now we consider the even and odd parts of polynomials separately. Put $\varepsilon=\left(\begin{array}{cc}-1 & 0 \\ 0 & 1\end{array}\right)$. By the identity

$$
\varepsilon(1-T)(1+M)=-(1-T)(1+M) T^{-1} \varepsilon
$$

(every matrix identity is regarded projectively, i.e., as that in $\Gamma_{0}(2) /\{ \pm 1\}$ ), we see that if $P \in W_{k}$ then $P \mid(1 \pm \varepsilon) \in W_{k}$, and so we have the direct sum decomposition

$$
W_{k}=W_{k}^{+} \oplus W_{k}^{-},
$$

where $W_{k}^{+}$(resp. $W_{k}^{-}$) is the even (resp. odd) part of $W_{k}$ :

$$
W_{k}^{ \pm}:=\left\{P \in V_{k}|P| \varepsilon= \pm P \text { and } P \mid(1-T)(1+M)=0\right\} .
$$

We also denote by $r_{f}^{ \pm}(X)$ the even and odd part of $r_{f}(X)$,

$$
r_{f}^{ \pm}(X):=\frac{1}{2} r_{f}(X) \mid(1 \pm \varepsilon)
$$

and by $r^{ \pm}$the map

$$
r^{ \pm}: S_{k}(2) \ni f \longmapsto r_{f}^{ \pm}(X) \in W_{k}^{ \pm} \otimes \mathbf{C} .
$$

For the space $W_{k}^{+}$of even polynomials, we have two obvious elements 1 and $X^{k-2}$. This is clear for 1 because $1 \mid(1-T)=0$. For $X^{k-2}$, we note the identity

$$
(1-T)(1+M)=(1-T M)(1+M) \quad\left(\text { because } M^{2}=1\right)
$$

and $T M=\left(\begin{array}{ll}1 & 0 \\ 2 & 1\end{array}\right)$ and thus $X^{k-2} \mid T M=X^{k-2}$. Hence, we have the decomposition

$$
W_{k}^{+}=\mathbf{Q} \cdot 1 \oplus \mathbf{Q} \cdot X^{k-2} \oplus W_{k}^{+, 0},
$$

where

$$
W_{k}^{+, 0}:=\left\{P(X) \in V_{k}\left|P(X)=\sum_{\substack{i=2 \\ \text { even }}}^{k-4} a_{i} X^{i}, P\right|(1-T)(1+M)=0\right\}
$$

Let $r^{+, 0}$ be the map $S_{k}(2) \rightarrow W_{k}^{+, 0}$ obtained by the composition of $r^{+}$and the natural projection $W_{k}^{+} \rightarrow W_{k}^{+, 0}$. From the works of Imamoḡlu-Kohnen [8] and FukuharaYang [6], we obtain the following

Theorem 4 For even $k$, the two maps

$$
r^{+, 0}: S_{k}(2) \longrightarrow W_{k}^{+, 0} \otimes \mathbf{C} \quad \text { and } \quad r^{-}: S_{k}(2) \longrightarrow W_{k}^{-} \otimes \mathbf{C}
$$

are isomorphisms of vector spaces. 
Proof We know from [8] and [6] that both maps are injective. So all we have to show is the dimensions of the target spaces are equal to the dimension of $S_{k}(2)$, which is equal to $[k / 4]-1$. We only calculate the dimension of $W_{k}^{+, 0}$, since the other is similar and only the former is relevant to the subsequent story involving the double Eisenstein series. mial

Put $T^{\prime}=\left(\begin{array}{cc}1 & 0 \\ -1 & 1\end{array}\right)$. Obviously $P=0$ is equivalent to $P \mid T^{\prime}=0$. For an even polyno-

$$
P(X)=\sum_{\substack{2 \leq i \leq k-4 \\ i: \text { even }}} a_{i} X^{i}
$$

with no constant term and no $X^{k-2}$ term, we compute the condition $P \mid(1-T)(1+$ $M) T^{\prime}=0$ for $P$ being in $W_{k}^{+, 0}$. By

$$
(1-T)(1+M) T^{\prime}=T^{\prime}-T T^{\prime}-{ }^{t} T+M T^{\prime} \quad\left(T M T^{\prime}={ }^{t} T=\left(\begin{array}{ll}
1 & 0 \\
1 & 1
\end{array}\right)\right)
$$

and

$$
T T^{\prime}=\left(\begin{array}{cc}
0 & 1 \\
-1 & 1
\end{array}\right), M T^{\prime}=\left(\begin{array}{cc}
0 & -1 \\
1 & 1
\end{array}\right)
$$

the condition becomes

$(-X+1)^{k-2}\left(P\left(\frac{X}{-X+1}\right)-P\left(\frac{1}{-X+1}\right)\right)-(X+1)^{k-2}\left(P\left(\frac{X}{X+1}\right)-P\left(\frac{-1}{X+1}\right)\right)=0$,

which is written as

$$
\sum_{\substack{2 \leq i \leq k-4 \\ i: \text { even }}} a_{i}\left(X^{i}-1\right)\left((-X+1)^{k-2-i}-(X+1)^{k-2-i}\right)=0 .
$$

Using binomial theorem, we can write this as

$$
2 \sum_{\substack{1 \leq j \leq k-3 \\
j: \text { odd }}}\left(\sum_{\substack{2 \leq i \leq k-4 \\
\text { i:even }}}\left(\left(\begin{array}{c}
i \\
j
\end{array}\right)-\left(\begin{array}{c}
i \\
k-2-j
\end{array}\right)\right) a_{k-2-i}\right) X^{j}=0 .
$$

Therefore, the space $W_{k}^{+, 0}$ is the set of polynomials

$$
P(X)=\sum_{\substack{2 \leq i \leq k-4 \\ \text { i:even }}} a_{i} X^{i}
$$

whose (rational) coefficients satisfy a set of linear relations

$$
\sum_{\substack{2 \leq i \leq k-4 \\
i: \text { even }}}\left(\left(\begin{array}{c}
i \\
j
\end{array}\right)-\left(\begin{array}{c}
i \\
k-2-j
\end{array}\right)\right) a_{k-2-i}=0 \quad(j=1,3, \ldots, k-3) .
$$

Clearly the equations for $j$ and $k-2-j$ have just opposite sign, and so we have to look only at the equations for $j<k / 2-1$, the total number of equations being $[(k-2) / 4]$. But then for $i<j$ the coefficient of $a_{k-2-i}$ is zero, and the coefficient 
matrix is upper triangular with non-zero diagonals, or more explicitly, the equation can be solved to express $a_{k-4}, a_{k-6}, \ldots, a_{k-2[(k+2) / 4]}$ by the other coefficients. We thus see that the dimension of $W_{k}^{+, 0}$ is $k / 2-2-([(k+2) / 4]-1)=[k / 4]-1$, as desired.

Remark 1 We have not succeeded in characterizing the (codimension 2) image of $S_{k}(2)$ by $r^{+}$in $W_{k}^{+} \otimes \mathbf{C}$. It is expected that such a characterization as in [10] should exist.

Amazingly enough, the coefficient matrix in (22) appears exactly when we look at the imaginary part of the double Eisenstein series of level 2, which we are going to explain. We look at the imaginary part of $G_{r, k-r}^{\mathbf{0 o}}(\tau)$ (as $q$-series) for $r$ even. Let $\pi: \mathbf{C}[[q]] \longrightarrow \sqrt{-1} \mathbf{R}[[q]]$ be the natural projection to imaginary part (note that by imaginary part we mean the term in $\sqrt{-1} \mathbf{R}[[q]]$, not the coefficient of $\sqrt{-1}$ ). As Theorem 2 and Definition 1 (for $r=2$ ) shows, imaginary parts come from the terms with $\widetilde{\zeta} \mathbf{e}(p) g_{h}^{\mathbf{o}}(\tau)$ for odd $p$, and we have in matrix form

$$
\pi\left(\begin{array}{c}
G_{2, k-2}^{\mathbf{o o}}(q) \\
G_{4, k-4}^{\mathbf{0}}(q) \\
\vdots \\
G_{k-2,2}^{\mathbf{o o}}(q)
\end{array}\right)=Q_{k}\left(\begin{array}{c}
\widetilde{\zeta} \mathbf{e}(k-3) g_{3}^{\mathbf{0}}(q) \\
\widetilde{\zeta} \mathbf{e}(k-5) g_{5}^{\mathbf{0}}(q) \\
\vdots \\
\widetilde{\zeta} \mathbf{e}(3) g_{k-3}^{\mathbf{o}}(q)
\end{array}\right),
$$

where $Q_{k}$ is the $(k / 2-1) \times(k / 2-2)$ matrix given by

$$
\left.Q_{k}=\left(\left(\begin{array}{c}
2 j \\
2 i-1
\end{array}\right)-\left(\begin{array}{c}
2 j \\
k-2 i-1
\end{array}\right)\right)\right)_{\substack{1 \leq i \leq k / 2-1 \\
1 \leq j \leq k / 2-2}}
$$

This is exactly the coefficient matrix of (22)!

Let $\mathscr{D} \mathscr{E}_{k}$ be the $\mathbf{Q}$-vector space generated by $G_{r, k-r}^{\mathbf{o o}}(r=2,4, \ldots, k-2)$.

Theorem 5 Let $k \geq 4$ be a positive even integer.

1)

$$
\operatorname{dim} \mathscr{D} \mathscr{E}_{k}=\frac{k}{2}-1,
$$

so that the series $G_{r, k-r}^{\mathbf{o o}}(\tau)$ (r even) are linearly independent over $\mathbf{Q}$.

2) The space $\mathscr{D} \mathscr{E}_{k}$ contains $\mathbf{Q} \cdot(2 \pi i)^{-k} G_{k}^{(i \infty)}(\tau) \oplus S_{k}^{\mathbf{Q}}(2)$, where $S_{k}^{\mathbf{Q}}(2)$ is the space of cusp forms on $\Gamma_{0}(2)$ having rational Fourier coefficients.

Proof We first prove 2). By Theorem 3, we see the map

$$
\begin{aligned}
& Z_{r, s}^{\mathbf{e o}} \mapsto G_{s, r}^{\mathbf{e o}}(q), Z_{r, s}^{\mathbf{o e}} \mapsto G_{r, s}^{\mathbf{o e}}(q), Z_{r, s}^{\mathbf{o o}} \mapsto G_{r, s}^{\mathbf{o o}}(q), Z_{k}^{\mathbf{0}} \mapsto G_{k}^{\mathbf{o}}(q), \\
& P_{r, s}^{\mathbf{o e}} \mapsto G_{r}^{\mathbf{o}}(q) G_{s}^{\mathbf{e}}(q)+\frac{1}{4}\left(\delta_{r, 2} \bar{g}_{s}^{\mathbf{e}}(q)+\delta_{s, 2} \bar{g}_{r}^{\mathbf{o}}(q)\right), \\
& P_{r, s}^{\mathbf{o o}} \mapsto G_{r}^{\mathbf{o}}(q) G_{s}^{\mathbf{o}}(q)+\frac{1}{4}\left(\delta_{r, 2} \bar{g}_{s}^{\mathbf{o}}(q)+\delta_{s, 2} \bar{g}_{r}^{\mathbf{o}}(q)\right)
\end{aligned}
$$


gives a realization of the space $\mathscr{D} \mathscr{Z}_{k}$ in $\mathscr{D} \mathscr{E}_{k}$. Then, the first part of Theorem 1 ensures that the space $\mathscr{D}_{\mathscr{E}}$ contains $G_{k}^{\mathbf{o}}(q)=(2 \pi i)^{-k} G_{k}^{(i \infty)}(\tau)$, and by Theorem 3 we see that $G_{r}^{\mathbf{o}}(q) G_{s}^{\mathbf{e}}(q)$ and $G_{r}^{\mathbf{o}}(q) G_{s}^{\mathbf{o}}(q)(r+s=k, r, s>2)$ are contained in $\mathscr{D}_{\mathscr{E}_{k}}$. Because of the relation

$$
(2 \pi i)^{-k} G_{r}^{(0)}(\tau) G_{s}^{(i \infty)}(\tau)=\left(2^{r}-1\right) G_{r}^{\mathbf{e}}(q) G_{s}^{\mathbf{o}}(q)-G_{r}^{\mathbf{o}}(q) G_{s}^{\mathbf{o}}(q) \quad\left(q=e^{2 \pi i \tau}\right)
$$

and the fact shown by Imamoḡlu and Kohnen in [8] that these cusp forms $G_{r}^{(0)}(\tau) G_{s}^{(i \infty)}(\tau)$ generate the space $S_{k}(2)$, we obtain the assertion 2).

For 1), first we note by definition the inequality

$$
\operatorname{dim} \mathscr{D}_{k} \leq \frac{k}{2}-1
$$

Since elements in $\mathbf{Q} \cdot(2 \pi i)^{-k} G_{k}^{(i \infty)}(\tau) \oplus S_{k}^{\mathbf{Q}}(2)$ have no imaginary parts, they sit in the kernel of the projection $\pi$ from $\mathscr{D}_{k}$ to $\sqrt{-1} \mathbf{R}[[q]]$, thus

$$
\operatorname{dim} \operatorname{ker} \pi \geq 1+\operatorname{dim} S_{k}(2)=\left[\frac{k}{4}\right] .
$$

As for the dimension of the image of $\pi$, we see that it is equal to the rank of the matrix $Q_{k}$ because the series $g_{3}^{\mathbf{0}}(q), g_{5}^{\mathbf{0}}(q), \ldots, g_{k-3}^{\mathbf{0}}(q)$ are linearly independent over C. This can be seen as follows. For an odd prime $p$, the coefficient of $q^{p}$ in $g_{r}^{\mathbf{o}}(q)$ is $1+p^{r-1}$ times a constant independent of $p$. Hence by picking distinct odd prime numbers $p_{3}, p_{5}, \ldots, p_{k-5}$ and looking at the coefficients of $q, q^{p_{3}}, q^{p_{5}}, \ldots, q^{p_{k-5}}$ in $g_{3}^{\mathbf{o}}(q), g_{5}^{\mathbf{o}}(q), \ldots, g_{k-3}^{\mathbf{o}}(q)$, we see the desired linear independence because the coefficient matrix is essentially the (non-vanishing) Vandermonde determinant. We thus have

$$
\operatorname{dimim} \pi=\operatorname{rank} Q_{k}=\left[\frac{k+2}{4}\right]-1
$$

and therefore

$$
\operatorname{dim} \mathscr{D}_{k} \geq\left[\frac{k}{4}\right]+\left[\frac{k+2}{4}\right]-1=\frac{k}{2}-1 .
$$

Therefore we conclude

$$
\operatorname{dim} \mathscr{D}_{k}=\frac{k}{2}-1
$$

and also

$$
\operatorname{ker} \pi=\mathbf{Q} \cdot(2 \pi i)^{-k} G_{k}^{(i \infty)}(\tau) \oplus S_{k}^{\mathbf{Q}}(2)
$$

Corollary 1 For an even integer $k>2$, we have

$$
\operatorname{dim}\left\langle\zeta^{\mathbf{o o}}(2 r, k-2 r) \mid 1 \leq r \leq k / 2-1\right\rangle_{\mathbf{Q}} \leq \frac{k}{2}-1-\operatorname{dim} S_{k}(2) .
$$


Proof By taking the constant term of the $q$-series, we obtain the surjective map

$$
\mu: \mathscr{D} \mathscr{E}_{k} \longrightarrow\left\langle\zeta^{\mathbf{0 o}}(2 r, k-2 r) \mid 1 \leq r \leq k / 2-1\right\rangle_{\mathbf{Q}}
$$

By the theorem, the kernel of $\mu$ contains the space $S_{k}^{\mathbf{Q}}(2)$ and hence we obtain the corollary.

Remark 2 The above corollary says that among the $k / 2-1$ numbers $\zeta^{\mathbf{0 o}}$ (even, even) there are at least $\operatorname{dim} S_{k}(2)$ linear relations. It seems that the $k / 2-1$ numbers $\zeta^{\mathbf{0 o}}($ odd, odd $)$ are linearly independent over $\mathbf{Q}$, and the total space

$$
\left\langle\zeta^{\mathbf{o o}}(r, k-r) \mid 2 \leq r \leq k-1\right\rangle_{\mathbf{Q}}
$$

is spanned by $\zeta^{\mathbf{0 o}}(o d d, o d d)$ and $\zeta(k)$. (Recall the sum formula in Theorem 1, so that $\zeta(k)$ is contained in the above space.) The conjectural dimension of this space is thus $k / 2$. We also conjecture that the space of usual double zeta values of even weight $k$ is contained in the space spanned by $\zeta^{\mathbf{0 o}}(r, k-r)$ except $\zeta^{\mathbf{0}}(k-1,1)$ :

$$
\langle\zeta(r, k-r) \mid 2 \leq r \leq k-1\rangle_{\mathbf{Q}} \subset\left\langle\zeta^{\mathbf{o o}}(r, k-r) \mid 2 \leq r \leq k-2\right\rangle_{\mathbf{Q}}
$$

and that the space $\left\langle\zeta^{\mathbf{0 o}}(2 r, k-2 r) \mid 1 \leq r \leq k / 2-1\right\rangle_{\mathbf{Q}}$ is contained in the usual double zeta space:

$$
\left\langle\zeta^{\mathbf{0 o}}(2 r, k-2 r) \mid 1 \leq r \leq k / 2-1\right\rangle_{\mathbf{Q}} \subset\langle\zeta(r, k-r) \mid 2 \leq r \leq k-1\rangle_{\mathbf{Q}} .
$$

When $k$ is odd, we can prove that every $\zeta^{\mathbf{0 o}}(r, k-r)$ except $\zeta^{\mathbf{0}}(k-1,1)$ is a linear combination of $\zeta(r, k-r)$ :

$$
\left\langle\zeta^{\mathbf{o o}}(r, k-r) \mid 2 \leq r \leq k-2\right\rangle_{\mathbf{Q}} \subset\langle\zeta(r, k-r) \mid 2 \leq r \leq k-1\rangle_{\mathbf{Q}} .
$$

To prove this we use the identity of Y. Komori, K. Matsumoto, and H. Tsumura ([11])

$$
\begin{aligned}
(1+ & \left.(-1)^{r}\right) \zeta_{2}(r, s)+\left(1+(-1)^{s}\right) \zeta_{2}(s, r) \\
& =\sum_{\substack{i=0 \\
\text { even }}}^{k-3} 2^{-k+i+1}\left(\left(\begin{array}{c}
k-i-1 \\
r-1
\end{array}\right)+\left(\begin{array}{c}
k-i-1 \\
s-1
\end{array}\right)\right) \zeta(i) \zeta(k-i)-\zeta(k),
\end{aligned}
$$

valid when $r, s \geq 2$ and $r+s=k$ : odd, where

$$
\zeta_{2}(r, s)=\sum_{m, n \geq 1} \frac{1}{(m+2 n)^{r} m^{s}}=\zeta^{\mathbf{o o}}(r, s)+\zeta^{\mathbf{e e}}(r, s) .
$$

For $\zeta^{\mathbf{0 o}}(k-1,1)$, it seems we need $(\log 2) \zeta(k-1)$ other than usual double zeta values, but we have not proved this. 


\subsection{Proof of Theorem 3}

As in [7], we prove Theorem 3 by dividing it into three parts: the constant term, the imaginary part, and the combinatorial part. The double shuffle relation of the constant term is nothing but that of double zeta values, namely Proposition 1 and its regularization. As for the imaginary part, the assertion is as follows.

Lemma 3 For each integer $k>2$, we define generating functions $\mathscr{I}_{k}^{\mathbf{e o}}(X, Y), \mathscr{J}_{k}^{\mathbf{o e}}(X, Y)$, $\mathscr{I}_{k}^{\mathbf{0 o}}(X, Y)$ by

$$
\begin{aligned}
\mathscr{I}_{k}^{\mathbf{e o}}(X, Y) & :=\sum_{r+s=k} I_{r, s}^{\mathbf{e}} X^{r-1} Y^{s-1}, \mathscr{I}_{k}^{\mathbf{o e}}(X, Y):=\sum_{r+s=k} I_{r, S}^{\mathbf{o e}} X^{r-1} Y^{s-1}, \\
\mathscr{I}_{k}^{\mathbf{0 o}}(X, Y) & :=\sum_{r+s=k} I_{r, s}^{\mathbf{0 o}} X^{r-1} Y^{s-1} .
\end{aligned}
$$

Then we have

$$
\begin{aligned}
\sum_{\substack{p+h=k \\
p: \text { odd }}}\left(X^{h-1} Y^{p-1}+X^{p-1} Y^{h-1}\right) \tilde{\zeta}^{\mathbf{o}}(p) \bar{g}_{h}^{\mathbf{0}}(q) & =\mathscr{I}_{k}^{\mathbf{0 o}}(X, Y)+\mathscr{I}_{k}^{\mathbf{0 o}}(Y, X) \\
= & \mathscr{I}_{k}^{\mathbf{e o}}(X+Y, X)+\mathscr{I}_{k}^{\mathbf{e}}(X+Y, Y), \\
\sum_{\substack{p+h=k \\
p: \text { odd }}}\left(X^{h-1} Y^{p-1} \widetilde{\zeta}^{\mathbf{e}}(p) g_{h}^{\mathbf{o}}(q)+X^{p-1} Y^{h-1} \widetilde{\zeta}^{\mathbf{o}}(p) \bar{g}_{h}^{\mathbf{e}}(q)\right) & =\mathscr{I}_{k}^{\mathbf{o e}}(X, Y)+\mathscr{I}_{k}^{\mathbf{e o}}(Y, X) \\
& =\mathscr{I}_{k}^{\mathbf{o e}}(X+Y, X)+\mathscr{I}_{k}^{\mathbf{o o}}(X+Y, Y),
\end{aligned}
$$

where $\bar{g}_{h}^{\mathbf{o}}(q)$ and $\bar{g}_{h}^{\mathbf{e}}(q)$ are the series defined in (18).

Proof By definition, each generating function can be given as

$$
\begin{aligned}
\mathscr{I}_{k}^{\mathbf{e}}(X, Y)= & \sum_{\substack{p+h=k \\
p: \text { odd }}}\left(X^{h-1} Y^{p-1}-X^{h-1}(X-Y)^{p-1}\right) \widetilde{\zeta}^{\mathbf{o}}(p) g_{h}^{\mathbf{e}}(q) \\
& +\sum_{\substack{p+h=k \\
p: \text { odd }}} Y^{h-1}(X-Y)^{p-1} \widetilde{\zeta} \mathbf{o}(p) g_{h}^{\mathbf{o}}(q), \\
\mathscr{I}_{k}^{\mathbf{o}}(X, Y)= & \sum_{\substack{p+h=k \\
p: o d d}} X^{h-1} Y^{p-1} \widetilde{\zeta}^{\mathbf{e}}(p) g_{h}^{\mathbf{o}}(q)-\sum_{p+h=k} X^{h-1}(X-Y)^{p-1} \widetilde{\zeta}^{\mathbf{o}}(p) g_{h}^{\mathbf{o}}(q) \\
& +\sum_{\substack{p+h=k \\
p: o d d}} Y^{h-1}(Y-X)^{p-1} \widetilde{\zeta} \mathbf{o}(p) g_{h}^{\mathbf{e}}(q), \\
\mathscr{I}_{k}^{\mathbf{o o}}(X, Y)= & \sum_{\substack{p+h=k \\
p: \text { odd }}}\left(Y^{h-1}(Y-X)^{p-1}-X^{h-1}(Y-X)^{p-1}\right) \widetilde{\zeta} \mathbf{e}(p) g_{h}^{\mathbf{o}}(q) \\
& +\sum_{\substack{p+h=k \\
p: o d d}} X^{h-1} Y^{p-1} \widetilde{\zeta}^{\mathbf{o}}(p) g_{h}^{\mathbf{o}}(q) .
\end{aligned}
$$


The lemma follows from these by a simple calculation using binomial theorem and we omit the details.

For the computation of the combinatorial part, we prepare the generating functions as follows.

$$
\begin{aligned}
& \beta(X):=\sum_{p>0} \beta_{p} X^{p-1}=\frac{1}{2}\left(\frac{1}{X}-\frac{1}{e^{X}-1}\right), \\
& \beta^{\mathbf{e}}(X):=\sum_{p>0} \beta_{p}^{\mathbf{e}} X^{p-1}=\frac{1}{4}\left(\frac{2}{X}-\frac{1}{e^{\frac{X}{2}}-1}\right), \\
& \beta^{\mathbf{o}}(X):=\sum_{p>0} \beta_{p}^{\mathbf{o}} X^{p-1}=\frac{1}{4} \frac{1}{e^{\frac{X}{2}}+1}, \\
& g^{\mathbf{e}}(X):=\sum_{p>0} g_{p}^{\mathbf{e}}(\tau) X^{p-1}=-\frac{1}{2} \sum_{u>0} e^{\frac{-u X}{2}} \cdot \frac{q^{u}}{1-q^{u}}, \\
& g^{\mathbf{o}}(X):=\sum_{p>0} g_{p}^{\mathbf{o}}(\tau) X^{p-1}=-\frac{1}{2} \sum_{u>0}(-1)^{u} e^{\frac{-u X}{2}} \cdot \frac{q^{u}}{1-q^{u}}, \\
& \bar{g}^{\mathbf{e}}(X):=\sum_{p>0} \bar{g}_{p}^{\mathbf{e}}(\tau) X^{p-1}=\frac{1}{2 X}\left(\sum_{u>0} e^{\frac{-u X}{2}} \cdot \frac{q^{u}}{\left(1-q^{u}\right)^{2}}-4 g_{2}^{\mathbf{e}}(\tau)\right), \\
& \bar{g}^{\mathbf{o}}(X):=\sum_{p>0} \bar{g}_{p}^{\mathbf{o}}(\tau) X^{p-1}=\frac{1}{2 X}\left(\sum_{u>0}(-1)^{u} e^{\frac{-u X}{2}} \cdot \frac{q^{u}}{\left(1-q^{u}\right)^{2}}-4 g_{2}^{\mathbf{o}}(\tau)\right), \\
& g^{\mathbf{e o}}(X, Y):=\sum_{r, s \geq 1} g_{r, s}^{\mathbf{e o}}(\tau) X^{r-1} Y^{s-1}=\frac{1}{4} \sum_{u, v>0}(-1)^{v} e^{-\frac{u X+v Y}{2}} \cdot \frac{q^{u}}{1-q^{u}} \cdot \frac{q^{u+v}}{1-q^{u+v}}, \\
& g^{\mathbf{o e}}(X, Y):=\sum_{r, s \geq 1} g_{r, s}^{\mathbf{o}}(\tau) X^{r-1} Y^{s-1}=\frac{1}{4} \sum_{u, v>0}(-1)^{u} e^{-\frac{u X+v Y}{2}} \cdot \frac{q^{u}}{1-q^{u}} \cdot \frac{q^{u+v}}{1-q^{u+v}}, \\
& g^{\mathbf{o o}}(X, Y):=\sum_{r, s \geq 1} g_{r, s}^{\mathbf{o o}}(\tau) X^{r-1} Y^{s-1}=\frac{1}{4} \sum_{u, v>0}(-1)^{u+v} e^{-\frac{u X+v Y}{2}} \cdot \frac{q^{u}}{1-q^{u}} \cdot \frac{q^{u+v}}{1-q^{u+v}} \\
& \beta^{\mathbf{e o}}(X, Y):=\sum_{r, s \geq 1} \beta_{r, s}^{\mathbf{e o}}(\tau) X^{r-1} Y^{s-1}=\beta^{\mathbf{o}}(Y) g^{\mathbf{e}}(X)-\beta^{\mathbf{o}}(X-Y)\left(g^{\mathbf{e}}(X)-g^{\mathbf{o}}(Y)\right), \\
& \beta^{\mathbf{o e}}(X, Y):=\sum_{r, s \geq 1} \beta_{r, s}^{\mathbf{o e}}(\tau) X^{r-1} Y^{s-1}=\beta^{\mathbf{e}}(Y) g^{\mathbf{o}}(X)-\beta^{\mathbf{o}}(X-Y)\left(g^{\mathbf{o}}(X)-g^{\mathbf{e}}(Y)\right), \\
& \beta^{\mathbf{o o}}(X, Y):=\sum_{r, s \geq 1} \beta_{r, s}^{\mathbf{o o}}(\tau) X^{r-1} Y^{s-1}=\beta^{\mathbf{o}}(Y) g^{\mathbf{o}}(X)-\beta^{\mathbf{e}}(X-Y)\left(g^{\mathbf{o}}(X)-g^{\mathbf{o}}(Y)\right),
\end{aligned}
$$




$$
\begin{aligned}
\varepsilon^{\mathbf{e}}(X, Y) & :=\sum_{r, s \geq 1} \varepsilon_{r, s}^{\mathbf{e}}(\tau) X^{r-1} Y^{s-1} \\
& =X \bar{g}^{\mathbf{0}}(Y)-Y \bar{g}^{\mathbf{o}}(Y)-\bar{g}_{0}^{\mathbf{0}}(\tau)+X \bar{g}^{\mathbf{e}}(X)+\bar{g}_{0}^{\mathbf{e}}(\tau)+g^{\mathbf{e}}(X)+\alpha_{1}, \\
\varepsilon^{\mathbf{0}}(X, Y) & :=\sum_{r, s \geq 1} \varepsilon_{r, s}^{\mathbf{o e}}(\tau) X^{r-1} Y^{s-1} \\
& =X \bar{g}^{\mathbf{e}}(Y)-Y \bar{g}^{\mathbf{e}}(Y)-\bar{g}_{0}^{\mathbf{e}}(\tau)+X \bar{g}^{\mathbf{0}}(X)+\bar{g}_{0}^{\mathbf{o}}(\tau)+g^{\mathbf{o}}(X)+\alpha_{2}, \\
\varepsilon^{\mathbf{0}}(X, Y) & :=\sum_{r, s \geq 1} \varepsilon_{r, s}^{\mathbf{0 o}}(\tau) X^{r-1} Y^{s-1}=X \bar{g}^{\mathbf{o}}(Y)-Y \bar{g}^{\mathbf{o}}(Y)+X \bar{g}^{\mathbf{o}}(X)+g^{\mathbf{o}}(X)+\alpha_{3},
\end{aligned}
$$

where $\alpha_{1}, \alpha_{2}$ and $\alpha_{3}$ are defined as in (19). Let

$$
\begin{aligned}
\mathscr{C}^{\mathbf{e}}(X, Y) & :=\sum_{r, s \geq 1} C_{r, S}^{\mathbf{e o}} X^{r-1} Y^{s-1}, \\
\mathscr{C}^{\mathbf{0}}(X, Y) & :=\sum_{r, s \geq 1} C_{r, S}^{\mathbf{o e}} X^{r-1} Y^{s-1}, \\
\mathscr{C}^{\mathbf{0}}(X, Y) & :=\sum_{r, s \geq 1} C_{r, S}^{\mathbf{o o}} X^{r-1} Y^{s-1} .
\end{aligned}
$$

Then by definition we have

$$
\begin{aligned}
& \mathscr{C}^{\mathbf{e}}(X, Y)=g^{\mathbf{e}}(X, Y)+\beta^{\mathbf{e}}(X, Y)+\frac{1}{4} \varepsilon^{\mathbf{e}}(X, Y), \\
& \mathscr{C}^{\mathbf{0}}(X, Y)=g^{\mathbf{o}}(X, Y)+\beta^{\mathbf{o e}}(X, Y)+\frac{1}{4} \varepsilon^{\mathbf{o}}(X, Y), \\
& \mathscr{C}^{\mathbf{0}}(X, Y)=g^{\mathbf{o}}(X, Y)+\beta^{\mathbf{o}}(X, Y)+\frac{1}{4} \varepsilon^{\mathbf{o}}(X, Y) .
\end{aligned}
$$

The double shuffle relations of the combinatorial double Eisenstein series are stated as

\section{Lemma 4 Put}

$$
\begin{aligned}
\mathscr{Q}^{\mathbf{e}}(X, Y) & :=g^{\mathbf{o}}(X) g^{\mathbf{e}}(Y)+\beta^{\mathbf{o}}(X) g^{\mathbf{e}}(Y)+\beta^{\mathbf{e}}(Y) g^{\mathbf{o}}(X)+\frac{1}{4}\left(X \bar{g}^{\mathbf{e}}(Y)+Y \bar{g}^{\mathbf{o}}(X)\right), \\
\mathscr{Q}^{\mathbf{o}}(X, Y) & :=g^{\mathbf{o}}(X) g^{\mathbf{o}}(Y)+\beta^{\mathbf{o}}(X) g^{\mathbf{o}}(Y)+\beta^{\mathbf{o}}(Y) g^{\mathbf{o}}(X)+\frac{1}{4}\left(X \bar{g}^{\mathbf{o}}(Y)+Y \bar{g}^{\mathbf{o}}(X)\right), \\
\mathscr{C}^{\mathbf{o}}(X) & :=g^{\mathbf{o}}(X)-\frac{\alpha_{3}}{2} \cdot X .
\end{aligned}
$$

Then we have

$$
\begin{aligned}
& \mathscr{Q}^{\mathbf{0}}(X, Y)=\mathscr{C}^{\mathbf{0}}(X, Y)+\mathscr{C}^{\mathbf{e}}(Y, X)=\mathscr{C}^{\mathbf{0}}(X+Y, Y)+\mathscr{C}^{\mathbf{0}}(X+Y, X), \\
& \mathscr{Q}^{\mathbf{0}}(X, Y)=\mathscr{C}^{\mathbf{0}}(X, Y)+\mathscr{C}^{\mathbf{0}}(Y, X)+\frac{\mathscr{C}^{\mathbf{o}}(X)-\mathscr{C}^{\mathbf{0}}(Y)}{X-Y}=\mathscr{C}^{\mathbf{e}}(X+Y, X)+\mathscr{C}^{\mathbf{e}}(X+Y, Y) .
\end{aligned}
$$

Proof Computations are parallel to those in [7], though tedious, and we omit the details.

The two lemmas and Proposition 1 complete the proof of Theorem 3. 
Appendix : The double Eisenstein series and the period polynomials in the case of $\mathrm{SL}_{2}(\mathbf{Z})$

In this appendix we briefly recall the relation described in [9] between the double Eisenstein series and modular forms for $\mathrm{SL}_{2}(\mathbf{Z})$.

The double Eisenstein series for $\mathrm{SL}_{2}(\mathbf{Z})$ was first defined and studied in [7]:

$$
G_{r, s}(\tau):=(2 \pi i)^{-r-s} \sum_{\substack{\lambda>\mu>0 \\ \lambda, \mu \in \mathbf{Z} \cdot \tau+\mathbf{Z}}} \frac{1}{\lambda^{r} \mu^{s}}=(2 \pi i)^{-r-s} \sum_{\substack{m \tau+n>m^{\prime} \tau+n^{\prime}>0 \\ m, n, m^{\prime}, n^{\prime} \in \mathbf{Z}}} \frac{1}{(m \tau+n)^{r}\left(m^{\prime} \tau+n^{\prime}\right)^{s}} .
$$

Its Fourier series is given there as

$$
G_{r, s}(\tau)=\widetilde{\zeta}(r, s)+g_{r, s}(q)+\sum_{\substack{p+h=k \\
p>1}}\left((-1)^{s}\left(\begin{array}{c}
p-1 \\
s-1
\end{array}\right)+(-1)^{p+r}\left(\begin{array}{c}
p-1 \\
r-1
\end{array}\right)+\delta_{p, s}\right) \tilde{\zeta}(p) g_{h}(q)
$$

where $\widetilde{\zeta}(r, s)=(2 \pi i)^{-r-s} \zeta(r, s), \widetilde{\zeta}(p)=(2 \pi i)^{-p} \zeta(p)$, and

$$
\begin{aligned}
g_{r, s}(q) & =\frac{(-1)^{r+s}}{(r-1) !(s-1) !} \sum_{\substack{m>n>0 \\
u, v>0}} u^{r-1} v^{s-1} q^{u m+v n} \\
g_{h}(q) & =\frac{(-1)^{h}}{(h-1) !} \sum_{u, m>0} u^{h-1} q^{u m}
\end{aligned}
$$

By extending the definition in the case of non-absolute convergence using $q$-series, we showed that the double Eisenstein series satisfy the double shuffle relations (in the form described in [7]), that the space of double Eisenstein series contains the space of modular forms on $\mathrm{SL}_{2}(\mathbf{Z})$, and made a connection to the period polynomial by looking at the imaginary parts of the $q$-expansions of $G_{r, s}(\tau)$. Specifically, the imaginary parts are given, like (23), by

$$
\pi\left(\begin{array}{c}
G_{2, k-2}(\tau) \\
G_{3, k-3}(\tau) \\
\vdots \\
G_{k-2,2}(\tau)
\end{array}\right)=Q_{k}^{(1)}\left(\begin{array}{c}
\tilde{\zeta}(k-3) g_{3}(q) \\
\widetilde{\zeta}(k-5) g_{5}(q) \\
\vdots \\
\widetilde{\zeta}(3) g_{k-3}(q)
\end{array}\right),
$$

where $Q_{k}^{(1)}$ is the $(k-3) \times(k / 2-2)$ matrix given by

$$
Q_{k}^{(1)}=\left((-1)^{i}\left(\begin{array}{c}
2 j \\
i
\end{array}\right)-(-1)^{i}\left(\begin{array}{c}
2 j \\
k-2-i
\end{array}\right)+\delta_{k-2-i, 2 j}\right) \underset{\substack{1 \leq i \leq k-3 \\
1 \leq j \leq k / 2-2}}{ } .
$$

Rather surprisingly, this contains exactly $Q_{k}$ as a minor. For example,

$$
Q_{12}^{(1)}=\left(\begin{array}{cccc}
-2 & -4 & -6 & -8 \\
1 & 6 & 15 & 28 \\
0 & -4 & -20 & -48 \\
0 & 1 & 15 & 42 \\
0 & 0 & 0 & 0 \\
0 & 0 & -14 & -42 \\
0 & 4 & 20 & 48 \\
0 & -6 & -15 & -27 \\
2 & 4 & 6 & 8
\end{array}\right), Q_{12}=\left(\begin{array}{cccc}
-2 & -4 & -6 & -8 \\
0 & -4 & -20 & -48 \\
0 & 0 & 0 & 0 \\
0 & 4 & 20 & 48 \\
2 & 4 & 6 & 8
\end{array}\right) .
$$

Precisely, the $i$-th row of $Q_{k}$ is the $2 i-1$-st row of $Q_{k}^{(1)}$.

The right kernel of $Q_{k}^{(1)}$ corresponds to the even period polynomials (without constant term) of weight $k$ for $\mathrm{SL}_{2}(\mathbf{Z})$, an example being ${ }^{t}(1,-3,3,-1)$ in the right kernel of $Q_{12}^{(1)}$ and the corresponding period 
polynomial $X^{8}-3 X^{6}+3 X^{4}-X^{2}$ of weight 12 . As in Corollary 1 , by looking at the constant term of the double Eisenstein series and by using the connection to the period polynomial just mentioned, we obtain the upper bound of the dimension of the space of double zeta values:

$$
\operatorname{dim}\langle\zeta(r, k-r) \mid 2 \leq r \leq k-1\rangle_{\mathbf{Q}} \leq \frac{k}{2}-1-\operatorname{dim} S_{k}(1)
$$

Also, elements in the left kernel of $Q_{k}^{(1)}$ produce expressions of modular forms in terms of double Eisenstein series. By comparing the Fourier coefficients, we obtain certain formulas for Fourier coefficients of modular forms. Let us look at some examples in weight 12.

As the simplest example, take $(0,0,0,0,1,0,0,0,0)$ in the left kernel of $Q_{12}^{(1)}$. This corresponds to the relation

$$
2^{7} \cdot 3 \cdot 5^{2} \cdot 691 G_{6,6}(\tau)=2^{9} \cdot 3^{2} \cdot 5^{2} \widetilde{G}_{12}(\tau)-\Delta(\tau),
$$

where $\Delta(\tau)=q \Pi_{n>0}\left(1-q^{n}\right)^{24}=\sum_{n>0} \tau(n) q^{n}$ is the famous cusp form of weight 12 . Comparing the coefficients of both sides, we obtain

$$
\tau(n)=\frac{2}{693} \sigma_{11}(n)+\frac{691}{2^{2} \cdot 3^{2} \cdot 7} \sigma_{5}(n)-\frac{691}{2^{2} \cdot 3^{2}} \sigma_{3}(n)+\frac{5 \cdot 691}{2 \cdot 3^{2} \cdot 11} \sigma_{1}(n)-\frac{2 \cdot 691}{3} \rho_{5,5}(n),
$$

where

$$
\rho_{k, l}(n):=\sum_{\substack{a+b=n \\ a, b>0}} \sum_{\substack{\frac{a}{u}>\frac{b}{v} \\ \text { v }}} u^{k} v^{l}
$$

Incidentally, the Ramanujan congruence

$$
\tau(n) \equiv \sigma_{11}(n) \quad(\bmod 691)
$$

is clearly seen from this.

Secondly take $(0,0,7,28,0,20,0,0,0)$, which gives the relation

$2^{7} \cdot 3^{2} \cdot 5 \cdot 7 \cdot 691 G_{4,8}(\tau)+2^{9} \cdot 3^{2} \cdot 5 \cdot 7 \cdot 691 G_{5,7}(\tau)+2^{9} \cdot 3^{2} \cdot 5^{2} \cdot 691 G_{7,5}(\tau)=2^{5} \cdot 3^{3} \cdot 5 \cdot 11 \cdot 149 \widetilde{G}_{12}(\tau)-\Delta(\tau)$

and the formula

$$
\begin{aligned}
\tau(n)= & \frac{149}{840} \sigma_{11}(n)-\frac{691}{180} \sigma_{7}(n)-\frac{11747}{126} \sigma_{5}(n)+\frac{173441}{360} \sigma_{3}(n) \\
& -\frac{3455}{9} \sigma_{1}(n)-\frac{2764}{3} \rho_{3,7}(n)-\frac{19348}{3} \rho_{4,6}(n)-\frac{13820}{3} \rho_{6,4}(n)
\end{aligned}
$$

We may take yet other vectors in the left kernel of $Q_{12}^{(1)}$ (the dimension is 6) and may deduce similar kind of formulas for $\tau(n)$.

Remark 3 Interestingly enough, the matrix $Q_{k}^{(1)}$ appears when we write "motivic" double zeta values in terms of certain basis elements $f_{3}, f_{5}, \ldots$ using coproduct structure described in F. Brown's recent important papers $[4,5]$. One of the present authors has found the same relation between triple Eisenstein series and motivic triple zeta values. Or a variant (minor matrix) of $Q_{k}^{(1)}$ appears in the work of S. Baumard and L. Schneps [3] on a relation of double zeta values and period polynomials. Each of these should be related with each other, but we have not figured out the exact relations.

\section{References}

1. Apostol, T.M.: Modular functions and Dirichlet series in number theory. Springer: Berlin Heidelberg New York, (1976)

2. Arakawa, T., Kaneko, M.: Multiple $L$-values. J. Math. Soc. Japan 56(4), 967-991 (2004)

3. Baumard, S., Schneps, L.: Period polynomial relations between double zeta values. preprint (2011)

4. Brown, F.: Mixed Tate motives over Z. Ann. Math. 175-2, 949-976 (2012) 
5. Brown, F.: On the decomposition of motivic multiple zeta values. "Galois-Teichmüller theory and Arithmetic Geometry", Advanced Studies in Pure Mathematics, 31-58 (2012)

6. Fukuhara, S., Yang, Y.: Period polynomials and explicit formulas for Hecke operators on $\Gamma_{0}(2)$. Math Proc. Cambridge Philos. Soc. 146(2), 321-350 (2009)

7. Gangl, H., Kaneko, M., Zagier, D.: Double zeta values and modular forms. Automorphic forms and Zeta functions. World Sci. Publ. 71-106 (2006)

8. Imamoḡlu, Ö., Kohnen, W.: Representations of integers as sums of an even number of squares. Math. Ann. 333(4), 815-829 (2005)

9. Kaneko, M.: Double zeta values and modular forms. Proceedings of the Japan-Korea joint seminar on Number Theory (Kuju, Japan) (H. K. Kim and Y. Taguchi, eds.), October 2004.

10. Kohnen, K., Zagier, D.: Modular forms with rational periods. In Modular forms (Durham, 1983), Ellis Horwood Ser. Math. Appl.: Statist. Oper. Res., pp 197-249. Horwood, Chichester, (1984)

11. Komori, Y., Matsumoto, K., Tsumura, H.: Multiple zeta values and zeta-functions of root systems. Proc. Japan Acad., 87, Ser. A 103-107 (2011)

12. Tasaka, K.: On a conjecture for representations of integers as sums of squares and double shuffle relations. preprint $(2011)$ 